\title{
EXISTENCE AND UNIFORM DECAY OF SOLUTIONS FOR A CLASS OF GENERALIZED PLATE-MEMBRANE-LIKE SYSTEMS
}

HAIHONG LIU AND NING SU

Received 19 December 2005; Accepted 2 May 2006

We study the global existence, uniqueness, and asymptotic behavior of solutions for a class of generalized plate-membrane-like systems with nonlinear damping and source acting both interior and on boundary.

Copyright (c) 2006 Hindawi Publishing Corporation. All rights reserved.

\section{Introduction}

In this article, we are interested in the following wave coupled system with nonlinear damping and source acting both interior and on boundary:

$$
\begin{gathered}
u^{\prime \prime}+\beta_{1} \Delta^{2} u-\Phi\left(\|\nabla u\|^{2},\|\nabla v\|^{2}\right) \Delta u-\beta_{2} \Delta u^{\prime}+a(u-v)+g_{1}\left(u^{\prime}\right)=h_{1} * J_{1}(\Delta u) \text { in } \Omega, t>0 \\
v^{\prime \prime}-\Phi\left(\|\nabla u\|^{2},\|\nabla v\|^{2}\right) \Delta v-\beta_{3} \Delta v^{\prime}+a(v-u)+g_{2}\left(v^{\prime}\right)=0 \quad \text { in } \Omega, t>0, \\
u=\frac{\partial u}{\partial v}=0 \quad \text { on } \partial \Omega, t>0, \\
v=0 \quad \text { on } \sigma, t>0, \\
\Phi\left(\|\nabla u\|^{2},\|\nabla v\|^{2}\right) \frac{\partial v}{\partial v}+\beta_{3} \frac{\partial v^{\prime}}{\partial v}+v+v^{\prime}+g\left(v^{\prime}\right)=h_{2} * J_{2}(v) \quad \text { on } \Gamma, t>0, \\
u(0, x)=u^{0}(x), \quad v(0, x)=v^{0}(x) \quad \text { in } \Omega, \\
u^{\prime}(0, x)=u^{1}(x), \quad v^{\prime}(0, x)=v^{1}(x) \quad \text { in } \Omega,
\end{gathered}
$$

where

$$
\begin{gathered}
h_{1} * J_{1}(\Delta u(t))=\int_{0}^{t} h_{1}(t-s) J_{1}(\Delta u(s)) d s, \\
h_{2} * J_{2}(v(t))=\int_{0}^{t} h_{2}(t-s) J_{2}(v(s)) d s,
\end{gathered}
$$

Hindawi Publishing Corporation

International Journal of Mathematics and Mathematical Sciences

Volume 2006, Article ID 83931, Pages 1-24

DOI 10.1155/IJMMS/2006/83931 
and $\beta_{i} \geq 0, i=1,2,3$ are constants, $\Omega$ is a bounded domain in $\mathbb{R}^{n}$ with $C^{2}$ boundary $\partial \Omega=\Gamma \cup \sigma$, mes $\{\sigma\} \neq 0$ and $\bar{\Gamma} \cap \bar{\sigma}=\varnothing, v$ is the outward unit normal vector, $u^{\prime}=d u / d t$, $u^{\prime \prime}=d^{2} u / d t^{2}, \ldots, \Delta^{2}$ is the biharmonic operator, and $\Phi(s)$ is $C^{1}$ class function like $1+s$. Moreover $g_{i}, h_{i}, J_{i}, i=1,2$, are functions satisfying some general assumptions.

From the physical background, when $\Phi(s)=1$ and $\beta_{2}=\beta_{3}=g_{1}=g_{2}=h_{1}=0,(1.1)$ and (1.2) reduce to the so-called Petrovsky equation and wave equation. They are simplified models of elastic plates, beams, vibrating strings, membranes or elastic materials, and so forth. As an example in engineering, rubber and rubber-like materials are used to absorb vibration or shield structures from vibration. When dimension $n=2$, Oniszczuk [13] studied the free transverse vibrations of an elastically connected rectangular platemembrane systems. This vibratory system model considered comprises a three-layered structure which is composed of a thin plate, a massless elastic layer modelled as a homogeneous Winkler-type foundation, and a parallel membrane stretched uniformly by suitable constant tensions applied at the edges. In this paper, we will consider a class of generalized plate-membrane-like system showed in (1.1)-(1.7), where $\Phi(s)=1+s$ appear known as the Kirchhoff-type equation which means we consider the extensible plate and membrane. In this physical aspect of Kirchhoff-type coefficient, we refer the reader to Choo and Chung [6] and $\mathrm{Ma}[12]$.

So far, many authors have considered the similar viscoelastic problems with damping and source term acting in the domain or on the boundary. Among them in case of single equation, we can cite Cavalcanti et al. [5] and Aassila et al. [2]. We also mention the works connected with viscoelastic effects such as Jiang and Muñoz Rivera [9]. Cavalcanti et al. [4] studied the existence and exponential decay for a Kirchhoff-carrier model with viscosity. Furthermore, related to blowup of solutions in the domain we can cite the work of Vitillaro [16] and Georgiev and Todorova [7]. In the case of dissipative coupled systems of the wave equations, Aassila [1] studied a linear system of compactly coupled wave equations with nonlinear boundary frictional damping in both equations. He obtained the decay estimates of energy of the corresponding solutions. Some other coupled systems with internal damping or with another coupling type can be found in $[10,15]$.

In the case of $\Phi(s)=1, \beta_{2}=\beta_{3}=h_{1}=h_{2}=0$, and Dirichlet boundary condition, Guesmia [8] studied the so-called nonlinear coupled wave equation and Petrovsky system. Moreover, when $\Phi(s)=1, \beta_{2}=\beta_{3}=h_{1}=0$, and $J_{2}$ has a concrete form, Bae [3] studied the similar systems to systems (1.1)-(1.7).

In this paper, we will research existence, uniqueness, and uniform decay of solutions of systems (1.1)-(1.7). A distinctive character in this paper is to deal with the difficulties appearing in the proof of existence and exponential decay when Kirchhoff-type coefficients occur and $h_{1} * J_{1}(\Delta u), h_{2} * J_{2}(v)$ appear as the internal and external sources, respectively. Meanwhile, we use the generalized assumption and remove some restriction on $h_{2}$ compared to [3]. In order to obtain the exponential decay of the energy, we make use of the perturbed energy method, for instance Komornik and Zuazua [11].

Our paper is organized as follows: in Section 2, we give out an assumption and state the main result. In Section 3, we exploit the Faedo-Galerkin's approximation, a priori estimates, and compactness arguments to obtain the existence of solutions. In Section 4, 
uniqueness is proved under additional assumptions. In Section 5, the exponential decay of solution is obtained by using the perturbed energy method.

\section{Assumptions and main results}

Throughout this paper, use the following notation:

$$
\begin{aligned}
(u, v) & =\int_{\Omega} u(x) v(x) d x, & \|u\|^{2} & =\int_{\Omega}|u(x)|^{2} d x \\
(u, v)_{\Gamma} & =\int_{\Gamma} u(x) v(x) d \Gamma, & \|u\|_{\Gamma}^{2} & =\int_{\Gamma}|u(x)|^{2} d \Gamma
\end{aligned}
$$

and denote $V:=\left\{v \in H^{1}(\Omega):\left.v\right|_{\sigma}=0\right\}$, a closed subspace of $H^{1}(\Omega)$.

Furthermore, we pointed out some facts to be used later:

$$
\begin{gathered}
\|v\|^{2} \leq \mu_{1}\|\Delta v\|^{2}, \quad v \in H_{0}^{2}(\Omega) \\
\|v\|^{2} \leq \mu_{2}\|\nabla v\|^{2}, \quad\|v\|_{\Gamma}^{2} \leq \mu_{3}\|\nabla v\|^{2}, \quad v \in H_{0}^{1}(\Omega) .
\end{gathered}
$$

Now we state the main hypothesis in this paper.

(A.1) Assumption on initial condition. Let $u^{0} \in H^{4}(\Omega) \cap H_{0}^{2}(\Omega), u^{1} \in H^{2}(\Omega) \cap H_{0}^{1}(\Omega)$, and $v^{0}, v^{1} \in V \cap H^{2}(\Omega)$ verifying the compatibility condition

$$
\Phi\left(\left\|\nabla u^{0}\right\|^{2},\left\|\nabla v^{0}\right\|^{2}\right) \frac{\partial v^{0}}{\partial \nu}+\beta_{3} \frac{\partial v^{1}}{\partial \nu}+v^{0}+v^{1}+g\left(v^{1}\right)=0 \quad \text { on } \Gamma
$$

(A.2) Assumption on $g_{i}, i=1,2 . g_{i}: \mathbb{R} \rightarrow \mathbb{R}, i=1,2$, are nondecreasing $C^{1}$ functions satisfying $g_{i}(0)=0$, and there exist positive constants $\alpha_{1}, \alpha_{2}$ such that

$$
\alpha_{1}|s| \leq\left|g_{i}(s)\right| \leq \alpha_{2}|s| \quad \forall s \in \mathbb{R}, i=1,2
$$

(A.3) Assumption on $g . g: \mathbb{R} \rightarrow \mathbb{R}$ is a $C^{1}$ function and there exist positive constants $\alpha_{3}$, $\alpha_{4}, \alpha_{5}$ such that

$$
\begin{gathered}
\alpha_{3}|s|^{\rho} s \leq g(s) \leq \alpha_{4}|s|^{\rho} s \quad \forall s \in \mathbb{R}, \\
\alpha_{5}|s|^{\rho} \leq g^{\prime}(s) \quad \forall s \in \mathbb{R},
\end{gathered}
$$

where $\rho>0$.

(A.4) Assumption on $J_{1} . J_{1}: \mathbb{R} \rightarrow \mathbb{R}$ is a $C^{0}$ function verifying

$$
\left|J_{1}(s)\right| \leq M_{1}(1+|s|), \quad \forall s \in \mathbb{R}
$$

where $M_{1}$ is a positive constant. 
4 Existence and uniform decay of solutions

(A.5) Assumption on $J_{2} . J_{2}: \mathbb{R} \rightarrow \mathbb{R}$ is a $C^{0}$ function such that $J_{2}(0)=0$ and there exists a positive constant $M_{2}$ verifying

$$
\left|J_{2}(s)-J_{2}(t)\right| \leq M_{2}\left(|s|^{\gamma}+|t|^{\gamma}\right)|s-t| \quad \forall s, t \in \mathbb{R}
$$

where $\gamma>0$.

(A.6) Assumption on $h_{i}, i=1,2$. Assume that $h_{i} \in W^{1,1}(0, \infty) \cap W^{1, \infty}(0, \infty)$ are nonnegative functions verifying

$$
h_{i}(0)=0, \quad h_{i}^{\prime}(t) \leq-M_{3} h_{i}(t) \quad \forall t \geq t_{0}>0,
$$

where $M_{3}$ is a positive constant.

(A.7) Additional assumption on $a$ and $h_{i}, i=1,2$. Let $a<1 / \sqrt{\mu_{1} \mu_{2}}$ and let $h_{i}$ verify

$$
l_{1}:=a-\int_{0}^{\infty} h_{1}(s) d s>0, \quad l_{2}:=1-\int_{0}^{\infty} h_{2}(s) d s>0
$$

Next we define the energy $E(t)$

$$
\begin{aligned}
E(t)= & \frac{1}{2}\left(\left\|u^{\prime}(t)\right\|^{2}+\left\|v^{\prime}(t)\right\|^{2}+\|\nabla u(t)\|^{2}+\|\nabla v(t)\|^{2}+\|v(t)\|_{\Gamma}^{2}\right)+\frac{a}{2}\|u(t)\|^{2} \\
& +\frac{a}{2}\|v(t)\|^{2}+\frac{\beta_{1}}{2}\|\Delta u(t)\|^{2}+\frac{1}{4}\left(\|\nabla u(t)\|^{2}+\|\nabla v(t)\|^{2}\right)^{2}-\int_{\Omega} a u(t) v(t) d x
\end{aligned}
$$

Here, it is easy to see that the energy is nonnegative.

Theorem 2.1. Let Assumptions (A.1)-(A.6) hold, in which $\gamma$, $\rho$ satisfy $0<\gamma \leq \rho \leq 1 /(n-$ 2) if $n \geq 3, \gamma, \rho>0$ if $n=1,2$, then problem (1.1)-(1.7) has at least a solution $(u, v): \Omega \rightarrow \mathbb{R}^{2}$ such that

$$
\begin{gathered}
(u, v) \in L^{\infty}\left(0, \infty ; H_{0}^{2}(\Omega)\right) \times L^{\infty}(0, \infty ; V), \\
\left(u^{\prime}, v^{\prime}\right) \in L^{\infty}\left(0, \infty ; H_{0}^{2}(\Omega)\right) \times L^{\infty}(0, \infty ; V), \\
\left(u^{\prime \prime}, v^{\prime \prime}\right) \in L^{\infty}\left(0, \infty ; L^{2}(\Omega)\right) \times L^{\infty}\left(0, \infty ; L^{2}(\Omega)\right) .
\end{gathered}
$$

Furthermore, if $\gamma=\rho, M_{3} /(\gamma+2)>\left(\alpha_{3} / 2\left\|h_{2}\right\|_{\infty}\right)^{-1 /(\gamma+1)}$, and Assumption (A.7) holds, the following decay estimate is obtained:

$$
E(t) \leq C \exp (-\xi t) \quad \forall t \geq t_{0}
$$

where $C$ and $\xi$ are positive constants. In addition, if $J_{1}$ is global Lipschitz, then the solution is unique. 


\section{Existence of solutions}

The variational formulations of problem (1.1)-(1.7) are the following:

$$
\begin{aligned}
\left(u^{\prime \prime}, w\right) & +\beta_{1}(\Delta u, \Delta w)+\left(1+\|\nabla u\|^{2}+\|\nabla v\|^{2}\right)(\nabla u, \nabla w)+\beta_{2}\left(\nabla u^{\prime}, \nabla w\right) \\
& +(a(u-v), w)+\left(g_{1}\left(u^{\prime}\right), w\right) \\
= & \int_{0}^{t} h_{1}(t-s)\left(J_{1}(\Delta u(s)), w\right) d s, \quad w \in H_{0}^{2}(\Omega), \\
\left(v^{\prime \prime}, w\right)+ & \left(1+\|\nabla u\|^{2}+\|\nabla v\|^{2}\right)(\nabla v, \nabla w)+\beta_{3}\left(\nabla v^{\prime}, \nabla w\right)+(a(v-u), w) \\
& +\left(g_{2}\left(v^{\prime}\right), w\right)+(v, w)_{\Gamma}+\left(v^{\prime}, w\right)_{\Gamma}+\left(g\left(v^{\prime}\right), w\right)_{\Gamma} \\
= & \int_{0}^{t} h_{2}(t-s)\left(J_{2}(v(s)), w\right)_{\Gamma} d s, \quad w \in V .
\end{aligned}
$$

We will prove the existence of Theorem 2.1 in 5 steps.

Step 1 (approximate solutions). Let $\left\{w_{j}(x)\right\}_{j \in \mathbb{N}}$ be a base of $H_{0}^{2}(\Omega)$ which is orthonormal in $L^{2}(\Omega), V_{m}$ the subspace of $H_{0}^{2}(\Omega)$ generated by the first $m$ vectors of $\left\{w_{j}\right\}$. Let $\left\{\tilde{w}_{j}(x)\right\}_{j \in \mathbb{N}}$ be a base of $V \cap H^{2}(\Omega)$, orthonormal in $L^{2}(\Omega), \tilde{V}_{m}$ the subspace of $V \cap$ $H^{2}(\Omega)$ generated by the first $m$ vectors of $\left\{\tilde{w}_{j}\right\}$. We seek the approximate solutions

$$
u_{m}(t, x)=\sum_{j=1}^{m} g_{j m}(t) w_{j}(x), \quad v_{m}(t, x)=\sum_{j=1}^{m} \tilde{g}_{j m}(t) \widetilde{w}_{j}(x)
$$

of the following Cauchy problem:

$$
\begin{aligned}
\left(u_{m}^{\prime \prime}, w\right) & +\beta_{1}\left(\Delta u_{m}, \Delta w\right)+\left(1+\left\|\nabla u_{m}\right\|^{2}+\left\|\nabla v_{m}\right\|^{2}\right)\left(\nabla u_{m}, \nabla w\right)+\beta_{2}\left(\nabla u_{m}^{\prime}, \nabla w\right) \\
& +\left(a\left(u_{m}-v_{m}\right), w\right)+\left(g_{1}\left(u_{m}^{\prime}\right), w\right)=\int_{0}^{t} h_{1}(t-s)\left(J_{1}\left(\Delta u_{m}(s)\right), w\right) d s, \quad w \in V_{m} \\
\left(v_{m}^{\prime \prime}, w\right)+ & +\left(1+\left\|\nabla u_{m}\right\|^{2}+\left\|\nabla v_{m}\right\|^{2}\right)\left(\nabla v_{m}, \nabla w\right)+\beta_{3}\left(\nabla v_{m}^{\prime}, \nabla w\right)+\left(a\left(v_{m}-u_{m}\right), w\right) \\
& +\left(g_{2}\left(v_{m}^{\prime}\right), w\right)+\left(v_{m}, w\right)_{\Gamma}+\left(v_{m}^{\prime}, w\right)_{\Gamma}+\left(g\left(v_{m}^{\prime}\right), w\right)_{\Gamma} \\
= & \int_{0}^{t} h_{2}(t-s)\left(J_{2}\left(v_{m}(s)\right), w\right)_{\Gamma} d s, \quad w \in \tilde{V}_{m}
\end{aligned}
$$


6 Existence and uniform decay of solutions

satisfying the initial conditions

$$
\begin{aligned}
& u_{m}(0)=u_{m}^{0}=\sum_{j=1}^{m}\left(u^{0}, w_{j}\right) w_{j} \longrightarrow u^{0} \quad \text { in } H_{0}^{2}(\Omega), \\
& u_{m}^{\prime}(0)=u_{m}^{1}=\sum_{j=1}^{m}\left(u^{1}, w_{j}\right) w_{j} \longrightarrow u^{1} \quad \text { in } H_{0}^{2}(\Omega), \\
& v_{m}(0)=u_{m}^{0}=\sum_{j=1}^{m}\left(v^{0}, \tilde{w}_{j}\right) \tilde{w}_{j} \longrightarrow v^{0} \quad \text { in } V \cap H^{2}(\Omega), \\
& v_{m}^{\prime}(0)=v_{m}^{1}=\sum_{j=1}^{m}\left(v^{1}, \tilde{w}_{j}\right) \tilde{w}_{j} \longrightarrow v^{1} \quad \text { in } V \cap H^{2}(\Omega) .
\end{aligned}
$$

According to the ODE theory, we can solve the system (3.3)-(3.5) by Picard's iteration. Hence, this system has unique solution on interval $\left[0, T_{m}\right]$ for each $m$. The following estimates allow us to extend the solution to the closed interval $[0, T]$.

Step 2 (the first estimate). Replacing $w$ by $u_{m}^{\prime}(t)$ in (3.3) and by $v_{m}^{\prime}(t)$ in (3.4), respectively, and adding the results, we get

$$
\begin{aligned}
\frac{d}{d t}\left\{E_{m}(t)\right. & \left.+\frac{1}{\gamma+2}\left\|v_{m}(t)\right\|_{\gamma+2, \Gamma}^{\gamma+2}\right\}+\beta_{2}\left\|\nabla u_{m}^{\prime}(t)\right\|^{2}+\beta_{3}\left\|\nabla v_{m}^{\prime}(t)\right\|^{2}+\left\|v_{m}^{\prime}(t)\right\|_{\Gamma}^{2} \\
& +\left(g_{1}\left(u_{m}^{\prime}(t)\right), u_{m}^{\prime}(t)\right)+\left(g_{2}\left(v_{m}^{\prime}(t)\right), v_{m}^{\prime}(t)\right)+\left(g\left(v_{m}^{\prime}(t)\right), v_{m}^{\prime}(t)\right)_{\Gamma} \\
= & \int_{0}^{t} h_{2}(t-s)\left(J_{2}\left(v_{m}(s)\right), v_{m}^{\prime}(t)\right)_{\Gamma} d s+\int_{0}^{t} h_{1}(t-s)\left(J_{1}\left(\Delta u_{m}(s)\right), u_{m}^{\prime}(t)\right) d s \\
& +\left(\left|v_{m}(t)\right|^{\gamma} v_{m}(t), v_{m}^{\prime}(t)\right)_{\Gamma},
\end{aligned}
$$

where

$$
\begin{aligned}
E_{m}= & \frac{1}{2}\left(\left\|u_{m}^{\prime}(t)\right\|^{2}+\left\|v_{m}^{\prime}(t)\right\|^{2}+\left\|\nabla u_{m}(t)\right\|^{2}+\left\|\nabla v_{m}(t)\right\|^{2}+\left\|v_{m}(t)\right\|_{\Gamma}^{2}\right)+\frac{a}{2}\left\|u_{m}(t)\right\|^{2} \\
& +\frac{a}{2}\left\|v_{m}(t)\right\|^{2}+\frac{\beta_{1}}{2}\left\|\Delta u_{m}(t)\right\|^{2}+\frac{1}{4}\left(\left\|\nabla u_{m}(t)\right\|^{2}+\left\|\nabla v_{m}(t)\right\|^{2}\right)^{2}-\int_{\Omega} a u_{m}(t) v_{m}(t) d x .
\end{aligned}
$$

In the following we will use $C_{i}, i=0,1,2, \ldots$, to denote various positive constants which may be different in different places.

By the Assumption (A.4), we have

$$
\begin{aligned}
& \int_{0}^{t} h_{1}(t-s)\left(J_{1}\left(\Delta u_{m}(s)\right), u_{m}^{\prime}(t)\right) d s \\
& \quad \leq M_{1}\left\|h_{1}\right\|_{L^{1}(0, \infty)} \int_{0}^{t}\left(\left\|1+\left|\Delta u_{m}(s)\right|\right\|^{2}+\left\|u_{m}^{\prime}(t)\right\|^{2}\right) d t \\
& \quad \leq C_{1} \int_{0}^{t}\left\|\Delta u_{m}(s)\right\|^{2} d s+C_{2}\left\|u_{m}^{\prime}(t)\right\|^{2}+C_{3} .
\end{aligned}
$$


By (A.5), Hölder inequality, and Young's inequality, we have

$$
\begin{aligned}
\left(J_{2}\left(v_{m}(s)\right), v_{m}^{\prime}(t)\right)_{\Gamma} & \leq M_{2}\left\|v_{m}(s)\right\|_{\gamma+2, \Gamma}^{\gamma+1}\left\|v_{m}^{\prime}(t)\right\| \|_{\gamma+2, \Gamma} \\
& \leq C_{4}(\varepsilon)\left\|v_{m}(s)\right\|_{\gamma+2, \Gamma}^{\gamma+2}+\varepsilon\left\|v_{m}^{\prime}(t)\right\|_{\gamma+2, \Gamma}^{\gamma+2},
\end{aligned}
$$

where $\varepsilon>0$ is arbitrary.

From the embedding $L^{\rho+2}(\Gamma) \hookrightarrow L^{\gamma+2}(\Gamma)$ as $\rho \geq \gamma$ and (3.9), we obtain

$$
\begin{aligned}
& \int_{0}^{t} h_{2}(t-s)\left(J_{2}\left(v_{m}(s)\right), v_{m}^{\prime}(t)\right)_{\Gamma} d s \\
& \quad \leq\left\|h_{2}\right\|_{L^{1}(0, \infty)}\left(\int_{0}^{t} C_{4}(\varepsilon)\left\|v_{m}(s)\right\|_{\gamma+2, \Gamma}^{\gamma+2} d s+\varepsilon T\left\|v_{m}^{\prime}(t)\right\|_{\gamma+2, \Gamma}^{\gamma+2}\right) \\
& \quad \leq C_{5}(\varepsilon) \int_{0}^{t}\left\|v_{m}(s)\right\|_{\gamma+2, \Gamma}^{\gamma+2} d s+\varepsilon d\left\|v_{m}^{\prime}(t)\right\|_{\rho+2, \Gamma}^{\rho+2}+C_{6}(\varepsilon),
\end{aligned}
$$

where $d=b T\left\|h_{2}\right\|_{L^{1}(0, \infty)}$ and $b$ is the embedding constant of $L^{\rho+2}(\Gamma) \hookrightarrow L^{\gamma+2}(\Gamma)$.

Similarly, we have

$$
\begin{aligned}
\left(\left|v_{m}(t)\right|^{\gamma} v_{m}(t), v_{m}^{\prime}(t)\right)_{\Gamma} & \leq C_{7}(\varepsilon)\left\|v_{m}(t)\right\|_{\gamma+2, \Gamma}^{\gamma+2}+\varepsilon\left\|v_{m}^{\prime}(t)\right\|_{\gamma+2, \Gamma}^{\gamma+2} \\
& \leq C_{7}(\varepsilon)\left\|v_{m}(t)\right\|_{\gamma+2, \Gamma}^{\gamma+2}+C_{8}(\varepsilon)+\varepsilon\left\|v_{m}^{\prime}(t)\right\|_{\rho+2, \Gamma}^{\rho+2}
\end{aligned}
$$

Hence, from (3.8)-(3.11) we get

$$
\begin{aligned}
\frac{d}{d t}\left\{E_{m}(t)\right. & \left.+\frac{1}{\gamma+2}\left\|v_{m}(t)\right\|_{\gamma+2, \Gamma}^{\gamma+2}\right\}+\left(g_{1}\left(u_{m}^{\prime}(t)\right), u_{m}^{\prime}(t)\right)+\beta_{2}\left\|\nabla u_{m}^{\prime}(t)\right\|^{2} \\
& +\beta_{3}\left\|\nabla v_{m}^{\prime}(t)\right\|^{2}+\left\|v_{m}^{\prime}(t)\right\|_{\Gamma}^{2}+\left(g_{2}\left(v_{m}^{\prime}(t)\right), v_{m}^{\prime}(t)\right)+\left(\alpha_{3}-\varepsilon-\varepsilon d\right)\left\|v_{m}^{\prime}(t)\right\|_{\rho+2, \Gamma}^{\rho+2} \\
\leq & C_{1} \int_{0}^{t}\left\|\Delta u_{m}(s)\right\|^{2} d s+C_{2}\left\|u_{m}^{\prime}(t)\right\|^{2}+C_{5} \int_{0}^{t}\left\|v_{m}(s)\right\|_{\gamma+2, \Gamma}^{\gamma+2} d s+C_{7}\left\|v_{m}(t)\right\|_{\gamma+2, \Gamma}^{\gamma+2}+C_{9} .
\end{aligned}
$$

According to Assumption (A.2), we know that $\left(g_{1}\left(u_{m}^{\prime}(t)\right), u_{m}^{\prime}(t)\right) \geq 0$ and $\left(g_{2}\left(v_{m}^{\prime}(t)\right)\right.$, $\left.v_{m}^{\prime}(t)\right) \geq 0$. Moreover, we can choose $\varepsilon>0$ small enough such that $\alpha_{3}-\varepsilon-\varepsilon d=C_{0}>0$. 
8 Existence and uniform decay of solutions

Therefore, integrating (3.12) over $[0, t]$, we have

$$
\begin{aligned}
E_{m}(t)+ & \frac{1}{\gamma+2}\left\|v_{m}(t)\right\|_{\gamma+2, \Gamma}^{\gamma+2}+\beta_{2} \int_{0}^{t}\left\|\nabla u_{m}^{\prime}(s)\right\|^{2} d s+\beta_{3} \int_{0}^{t}\left\|\nabla v_{m}^{\prime}(s)\right\|^{2} d s \\
& +\int_{0}^{t}\left\|v_{m}^{\prime}(s)\right\|_{\Gamma}^{2} d s+C_{0} \int_{0}^{t}\left\|v_{m}^{\prime}(s)\right\|_{\rho+2, \Gamma}^{\rho+2} d s \\
\leq & C_{1} \iint_{0}^{t}\left\|\Delta u_{m}(s)\right\|^{2} d s d t+C_{2} \int_{0}^{t}\left\|u_{m}^{\prime}(s)\right\|^{2} d s+C_{5} \iint_{0}^{t}\left\|v_{m}(s)\right\|_{\gamma+2, \Gamma}^{\gamma+2} d s d t \\
& +C_{7} \int_{0}^{t}\left\|v_{m}(s)\right\|_{\gamma+2, \Gamma}^{\gamma+2} d s+C_{10} \\
\leq & C_{11} \int_{0}^{t}\left\|\Delta u_{m}(s)\right\|^{2} d s+C_{2} \int_{0}^{t}\left\|u_{m}^{\prime}(s)\right\|^{2} d s+C_{12} \int_{0}^{t}\left\|v_{m}(s)\right\|_{\gamma+2, \Gamma}^{\gamma+2} d s+C_{10} .
\end{aligned}
$$

Therefore, from (3.13) and using Gronwall's lemma we obtain the estimate

$$
\begin{aligned}
& \frac{1}{2}\left(\left\|u_{m}^{\prime}(t)\right\|^{2}+\left\|v_{m}^{\prime}(t)\right\|^{2}+\left\|\nabla u_{m}(t)\right\|^{2}+\left\|\nabla v_{m}(t)\right\|^{2}+\left\|v_{m}(t)\right\|_{\Gamma}^{2}\right) \\
& \quad+\frac{a}{2}\left\|u_{m}(t)\right\|^{2}+\frac{a}{2}\left\|v_{m}(t)\right\|^{2}+\frac{\beta_{1}}{2}\left\|\Delta u_{m}(t)\right\|^{2}+\frac{1}{4}\left(\left\|\nabla u_{m}(t)\right\|^{2}+\left\|\nabla v_{m}(t)\right\|^{2}\right)^{2} \\
& \quad-\int_{\Omega} a u_{m}(t) v_{m}(t) d x+\frac{1}{\gamma+2}\left\|v_{m}(t)\right\|_{\gamma+2, \Gamma}^{\gamma+2}+\beta_{2} \int_{0}^{t}\left\|\nabla u_{m}^{\prime}(s)\right\|^{2} d s \\
& +\beta_{3} \int_{0}^{t}\left\|\nabla v_{m}^{\prime}(s)\right\|^{2} d s+\int_{0}^{t}\left\|v_{m}^{\prime}(s)\right\|_{\Gamma}^{2} d s+C_{0} \int_{0}^{t}\left\|v_{m}^{\prime}(s)\right\|_{\rho+2, \Gamma}^{\rho+2} d s \leq L_{1},
\end{aligned}
$$

where $L_{1}>0$ is independent of $m$ and $t \in[0, T]$.

Step 3 (the second estimate). First we estimate the initial data $u_{m}^{\prime \prime}(0)$ and $v_{m}^{\prime \prime}(0)$ in the $L^{2}$-norm. Choosing $w=u_{m}^{\prime \prime}(0)$ in (3.3) and $w=v_{m}^{\prime \prime}(0)$ in (3.4), we obtain

$$
\begin{aligned}
\left\|u_{m}^{\prime \prime}(0)\right\|^{2} & +\beta_{1}\left(\Delta u^{0}, \Delta u_{m}^{\prime \prime}(0)\right)-\left(1+\left\|\nabla u_{m}(0)\right\|^{2}+\left\|\nabla v_{m}(0)\right\|^{2}\right)\left(\Delta u^{0}, u_{m}^{\prime \prime}(0)\right) \\
& +\beta_{2}\left(\nabla u^{1}, \nabla u_{m}^{\prime \prime}(0)\right)+\left(a u^{0}, u_{m}^{\prime \prime}(0)\right)-\left(a v^{0}, u_{m}^{\prime \prime}(0)\right)+\left(g_{1}\left(u^{1}\right), u_{m}^{\prime \prime}(0)\right)=0, \\
\left\|v_{m}^{\prime \prime}(0)\right\|^{2}-\left(1+\left\|\nabla u_{m}(0)\right\|^{2}+\left\|\nabla v_{m}(0)\right\|^{2}\right)\left(\Delta v^{0}, v_{m}^{\prime \prime}(0)\right)+\beta_{3}\left(\nabla v^{1}, \nabla v_{m}^{\prime \prime}(0)\right) & \\
& +\left(a v^{0}, v_{m}^{\prime \prime}(0)\right)-\left(a u^{0}, v_{m}^{\prime \prime}(0)\right)+\left(g_{2}\left(v^{1}\right), v_{m}^{\prime \prime}(0)\right)+\left(v^{0}, v_{m}^{\prime \prime}(0)\right)_{\Gamma} \\
& +\left(v^{1}, v_{m}^{\prime \prime}(0)\right)_{\Gamma}+\left(g\left(v^{1}\right), v_{m}^{\prime \prime}(0)\right)_{\Gamma}=0 .
\end{aligned}
$$

Hence from Assumption (A.1), (3.15), it is not hard to get

$$
\left\|u_{m}^{\prime \prime}(0)\right\|+\left\|v_{m}^{\prime \prime}(0)\right\| \leq L_{2}
$$

where $L_{2}$ is a positive constant independent of $m$. 
Differentiating (3.3) and (3.4), replacing $w$ by $u_{m}^{\prime \prime}(t)$ and $v_{m}^{\prime \prime}(t)$, respectively, then adding the results we get

$$
\begin{aligned}
\frac{d}{d t} F_{m}(t) & +\beta_{2}\left\|\nabla u_{m}^{\prime \prime}(t)\right\|^{2}+\beta_{3}\left\|\nabla v_{m}^{\prime \prime}(t)\right\|^{2}+\left\|v_{m}^{\prime \prime}(t)\right\|_{\Gamma}^{2}+\left(g_{1}^{\prime}\left(u_{m}^{\prime}(t)\right),\left|u_{m}^{\prime \prime}(t)\right|^{2}\right) \\
& +\left(g_{2}^{\prime}\left(v_{m}^{\prime}(t)\right),\left|v_{m}^{\prime \prime}(t)\right|^{2}\right)+\left(g^{\prime}\left(v_{m}^{\prime}(t)\right),\left|v_{m}^{\prime \prime}(t)\right|^{2}\right)_{\Gamma} \\
= & -\left(1+\left\|\nabla u_{m}(t)\right\|^{2}+\left\|\nabla v_{m}(t)\right\|^{2}\right)\left(\left(\nabla u_{m}^{\prime}(t), \nabla u_{m}^{\prime \prime}(t)\right)+\left(\nabla v_{m}^{\prime}(t), \nabla v_{m}^{\prime \prime}(t)\right)\right) \\
& -2\left(\left(\nabla u_{m}(t), \nabla u_{m}^{\prime}(t)\right)+\left(\nabla v_{m}(t), \nabla v_{m}^{\prime}(t)\right)\right) \\
& \times\left(\left(\nabla u_{m}(t), \nabla u_{m}^{\prime \prime}(t)\right)+\left(\nabla v_{m}(t), \nabla v_{m}^{\prime \prime}(t)\right)\right)+\int_{0}^{t} h_{2}^{\prime}(t-s)\left(J_{2}\left(v_{m}(s)\right), v_{m}^{\prime \prime}(t)\right)_{\Gamma} d s \\
& +\int_{0}^{t} h_{1}^{\prime}(t-s)\left(J_{1}\left(\Delta u_{m}(s)\right), u_{m}^{\prime \prime}(t)\right) d s \leq C_{13}(\varepsilon)\left(\left\|\nabla u_{m}^{\prime}(t)\right\|^{2}+\left\|\nabla v_{m}^{\prime}(t)\right\|^{2}\right) \\
& +\varepsilon\left(\left\|\nabla u_{m}^{\prime \prime}(t)\right\|^{2}+\left\|\nabla v_{m}^{\prime \prime}(t)\right\|^{2}\right)+\int_{0}^{t} h_{2}^{\prime}(t-s)\left(J_{2}\left(v_{m}(s)\right), v_{m}^{\prime \prime}(t)\right)_{\Gamma} d s \\
& +\int_{0}^{t} h_{1}^{\prime}(t-s)\left(J_{1}\left(\Delta u_{m}(s)\right), u_{m}^{\prime \prime}(t)\right) d s,
\end{aligned}
$$

where we denote that

$$
\begin{aligned}
F_{m}(t)= & \frac{1}{2}\left\|u_{m}^{\prime \prime}(t)\right\|^{2}+\frac{\beta_{1}}{2}\left\|\Delta u_{m}^{\prime}(t)\right\|^{2}+\frac{1}{2}\left\|v_{m}^{\prime \prime}(t)\right\|^{2}+\frac{1}{2}\left\|\nabla u_{m}^{\prime}(t)\right\|^{2} \\
& +\frac{a}{2}\left\|u_{m}^{\prime}(t)\right\|^{2}+\frac{a}{2}\left\|v_{m}^{\prime}(t)\right\|^{2}+\frac{1}{2}\left\|v_{m}^{\prime}(t)\right\|_{\Gamma}^{2}+\frac{1}{2}\left\|\nabla v_{m}^{\prime}(t)\right\|^{2} \\
& -\int_{\Omega} a u_{m}^{\prime}(t) v_{m}^{\prime}(t) d x .
\end{aligned}
$$

Noticing that $(\gamma+1) /(2 \gamma+2)+(1 / 2)=1$, Assumptions (A.5), (A.6), the embedding $H^{1}(\Omega) \hookrightarrow L^{q}(\Gamma)$ for $1 \leq q \leq(2 n-2) /(n-2)$, and the first estimate (3.14), we obtain

$$
\begin{aligned}
& \int_{0}^{t} h_{2}^{\prime}(t-s)\left(J_{2}\left(v_{m}(s)\right), v_{m}^{\prime \prime}(t)\right)_{\Gamma} d s \\
& \quad \leq M_{2}\left\|h_{2}^{\prime}\right\|_{L^{1}(0, \infty)} \int_{0}^{t}\left\{\left(\int_{\Gamma}\left|v_{m}(s)\right|^{2 \gamma+2} d \Gamma\right)^{(\gamma+1) /(2 \gamma+2)}\left(\int_{\Gamma}\left|v_{m}^{\prime \prime}(t)\right|^{2} d \Gamma\right)^{1 / 2}\right\} d s \\
& \quad \leq M_{2}\left\|h_{2}^{\prime}\right\|_{L^{1}(0, \infty)} \int_{0}^{t}\left\{C_{14}\left\|\nabla v_{m}(s)\right\|^{\gamma+1}\left\|v_{m}^{\prime \prime}(t)\right\|_{\Gamma}\right\} d s \\
& \quad \leq M_{2}\left\|h_{2}^{\prime}\right\|_{L^{1}(0, \infty)} \int_{0}^{t}\left\{\left(L_{1}\right)^{\gamma+1}\left\|v_{m}^{\prime \prime}(t)\right\|_{\Gamma}\right\} d s \\
& \leq C_{15}(\varepsilon)+\varepsilon\left\|v_{m}^{\prime \prime}(t)\right\|_{\Gamma}^{2} .
\end{aligned}
$$


Furthermore, noticing Assumption (A.4) and using Schwarz's inequality we obtain

$$
\begin{aligned}
\int_{0}^{t} h_{1}^{\prime}(t-s)\left(J_{1}\left(\Delta u_{m}(s)\right), u_{m}^{\prime \prime}(t)\right) d s & \leq C_{16}+C_{17}\left\|u_{m}^{\prime \prime}(t)\right\|^{2}+C_{18} \int_{0}^{t}\left\|\Delta u_{m}(s)\right\|^{2} d s \\
& \leq C_{19}+C_{17}\left\|u_{m}^{\prime \prime}(t)\right\|^{2} .
\end{aligned}
$$

Meanwhile, according to Assumption (A.2) we can deduce that $\left(g_{1}^{\prime}\left(u_{m}^{\prime}(t)\right),\left|u_{m}^{\prime \prime}(t)\right|^{2}\right)+$ $\left(g_{2}^{\prime}\left(v_{m}^{\prime}(t)\right),\left|v_{m}^{\prime \prime}(t)\right|^{2}\right) \geq 0$. Therefore, by (3.19), (3.20), integrating (3.17) over [0,t], making use of Gronwall lemma, and the first estimate, and (3.16), we get the second estimate

$$
\begin{aligned}
\frac{1}{2} \| u_{m}^{\prime \prime}(t) & \left\|^{2}+\frac{\beta_{1}}{2}\right\| \Delta u_{m}^{\prime}(t)\left\|^{2}+\frac{1}{2}\right\| v_{m}^{\prime \prime}(t)\left\|^{2}+\frac{1}{2}\right\| \nabla u_{m}^{\prime}(t)\left\|^{2}+\frac{a}{2}\right\| u_{m}^{\prime}(t) \|^{2} \\
& +\frac{a}{2}\left\|v_{m}^{\prime}(t)\right\|^{2}+\frac{1}{2}\left\|v_{m}^{\prime}(t)\right\|_{\Gamma}^{2}+\frac{1}{2}\left\|\nabla v_{m}^{\prime}(t)\right\|^{2}+\widetilde{\beta_{2}} \int_{0}^{t}\left\|\nabla u_{m}^{\prime \prime}(s)\right\|^{2} d s \\
& +\widetilde{\beta_{3}} \int_{0}^{t}\left\|\nabla v_{m}^{\prime \prime}(s)\right\|^{2} d s+\widetilde{\beta}_{4} \int_{0}^{t}\left\|v_{m}^{\prime \prime}(s)\right\|_{\Gamma}^{2} d s-\int_{\Omega} a u_{m}^{\prime}(t) v_{m}^{\prime}(t) d x \leq L_{3},
\end{aligned}
$$

where $\tilde{\beta}_{2}, \tilde{\beta}_{3}$, and $\tilde{\beta}_{4}$ are positive constants, $L_{3}>0$ is independent of $m$.

Step 4 (the third estimate). Let $m_{2} \geq m_{1}$ be two natural numbers and consider $y_{m}:=$ $u_{m_{2}}-u_{m_{1}}, z_{m}:=v_{m_{2}}-v_{m_{1}}$. From the system (3.4), we have

$$
\begin{aligned}
\left(z_{m}^{\prime \prime}, w\right) & +\left(\nabla z_{m}, \nabla w\right) \\
& +\left(\left[\left(\left\|\nabla u_{m_{2}}\right\|^{2}+\left\|\nabla v_{m_{2}}\right\|^{2}\right) \nabla v_{2}-\left(\left\|\nabla u_{m_{1}}\right\|^{2}+\left\|\nabla v_{m_{1}}\right\|^{2}\right) \nabla v_{1}\right], \nabla w\right) \\
& +\beta_{3}\left(\nabla z_{m}^{\prime}, \nabla w\right)+\left(a z_{m}, w\right)-\left(a y_{m}, w\right)+\left(\left[g_{2}\left(v_{m_{2}}^{\prime}\right)-g_{2}\left(v_{m_{1}}^{\prime}\right)\right], w\right) \\
& +\left(z_{m}, w\right)_{\Gamma}+\left(z_{m}^{\prime}, w\right)_{\Gamma}+\left(\left[g\left(v_{m_{2}}^{\prime}\right)-g\left(v_{m_{1}}^{\prime}\right)\right], w\right)_{\Gamma} \\
= & \int_{0}^{t} h_{2}(t-s)\left(\left[J_{2}\left(v_{m_{2}}(s)\right)-J_{2}\left(v_{m_{1}}(s)\right)\right], w(t)\right)_{\Gamma} d s, \quad w \in V .
\end{aligned}
$$

Substituting $w=z_{m}^{\prime}(t)$ in (3.22), observing that $g, g_{2}$ are nondecreasing, and using the previous estimates, we get

$$
\begin{aligned}
\frac{1}{2} \frac{d}{d t}\{ & \left.\left\|z_{m}^{\prime}(t)\right\|^{2}+\left\|\nabla z_{m}(t)\right\|^{2}+a\left\|z_{m}(t)\right\|^{2}+\left\|z_{m}(t)\right\|_{\Gamma}^{2}\right\} \\
& -\left(a y_{m}(t), z_{m}^{\prime}(t)\right)+\beta_{3}\left\|\nabla z_{m}^{\prime}(t)\right\|^{2}+\left\|z_{m}^{\prime}(t)\right\|_{\Gamma}^{2} \\
\leq & C_{20}\left\|\nabla z_{m}\right\|\left\|\nabla z_{m}^{\prime}\right\|+\int_{0}^{t} h_{2}(t-s)\left(\left[J_{2}\left(v_{m_{2}}(s)\right)-J_{2}\left(v_{m_{1}}(s)\right)\right], z_{m}^{\prime}(t)\right)_{\Gamma} d s \\
\leq & C_{21}(\eta)\left\|\nabla z_{m}\right\|^{2}+\eta\left\|\nabla z_{m}^{\prime}(t)\right\|^{2}+\eta\left\|z_{m}^{\prime}(t)\right\|_{\Gamma}^{2} \\
& +C_{22}(\eta) \int_{0}^{t}\left|h_{2}(t-s)\right|^{2}\left\|J_{2}\left(v_{m_{2}}(s)\right)-J_{2}\left(v_{m_{1}}(s)\right)\right\|_{\Gamma}^{2} d s,
\end{aligned}
$$

where $\eta>0$ is arbitrary. 
Moreover from Assumption (A.5) and the first estimate, we have

$$
\begin{aligned}
& \int_{0}^{t}\left|h_{2}(t-s)\right|^{2}\left\|J_{2}\left(v_{m_{2}}(s)\right)-J_{2}\left(v_{m_{1}}(s)\right)\right\|_{\Gamma}^{2} d s \\
& \quad \leq C_{23} \int_{0}^{t}\left(\left\|\nabla v_{m_{2}}(s)\right\|^{\gamma}+\left\|\nabla v_{m_{1}}(s)\right\|^{\gamma}\right)^{2}\left\|\nabla z_{m}(s)\right\|^{2} d s \\
& \quad \leq C_{24} \int_{0}^{t}\left\|\nabla z_{m}(s)\right\|^{2} d s .
\end{aligned}
$$

Hence integrating (3.23) over $(0, t)$ and using (3.24) we obtain

$$
\begin{gathered}
\frac{1}{2}\left[\left\|z_{m}^{\prime}(t)\right\|^{2}+\left\|\nabla z_{m}(t)\right\|^{2}+a\left\|z_{m}(t)\right\|^{2}+\left\|z_{m}(t)\right\|_{\Gamma}^{2}\right] \\
+\left(\beta_{3}-\eta\right) \int_{0}^{t}\left\|\nabla z_{m}^{\prime}(s)\right\|^{2} d s+(1-\eta) \int_{0}^{t}\left\|z_{m}^{\prime}(s)\right\|_{\Gamma}^{2} d s \\
\leq\left(C_{21}(\eta)+C_{24}\right) \int_{0}^{t}\left\|\nabla z_{m}(s)\right\|^{2} d s+a \int_{0}^{t}\left\|y_{m}(s)\right\|^{2} d s \\
+\int_{0}^{t}\left\|z_{m}^{\prime}(s)\right\|^{2} d s+C_{25}(T)\left(\left\|z_{1 m}\right\|^{2}+\left\|\nabla z_{0 m}\right\|^{2}\right) .
\end{gathered}
$$

Hence letting $\eta>0$ small enough, by the first estimate and using Gronwall's lemma of integral form (see [14]) we obtain that

$$
\begin{aligned}
& \left\|z_{m}^{\prime}(t)\right\|^{2}+\left\|\nabla z_{m}(t)\right\|^{2}+a\left\|z_{m}(t)\right\|^{2}+\left\|z_{m}(t)\right\|_{\Gamma}^{2} \\
& \quad \leq C_{26}(T)\left(\left\|z_{1 m}\right\|^{2}+\left\|\nabla z_{0 m}\right\|^{2}+\int_{0}^{T}\left\|y_{m}(s)\right\|^{2} d s\right) .
\end{aligned}
$$

Step 5 (passage to the limit). Above two estimates are sufficient to pass to the limit in the linear terms of problem (3.3), (3.4). In the following we will consider the nonlinear terms.

Due to the above estimates and using the regularity theory of elliptic boundary problem, we deduce that

$$
\begin{gathered}
\left\{u_{m}(t)\right\} \text { is bounded in } L^{2}\left(0, T ; H_{0}^{2}(\Omega)\right), \\
\left\{u_{m}^{\prime}(t)\right\} \text { is bounded in } L^{2}\left(0, T ; H_{0}^{2}(\Omega)\right), \\
\left\{u_{m}^{\prime \prime}(t)\right\} \text { is bounded in } L^{2}\left(0, T ; L^{2}(\Omega)\right), \\
\left\{v_{m}(t)\right\} \text { is bounded in } L^{2}(0, T ; V), \\
\left\{v_{m}^{\prime}(t)\right\} \text { is bounded in } L^{2}(0, T ; V), \\
\left\{v_{m}^{\prime \prime}(t)\right\} \text { is bounded in } L^{2}\left(0, T ; L^{2}(\Omega) \cap L^{2}(\Gamma)\right) .
\end{gathered}
$$

In the following, we will use the same notation to express the subsequences of $\left\{v_{m}(t)\right\}$ and $\left\{u_{m}(t)\right\}$. Considering the embedding $H^{1}(\Omega) \hookrightarrow L^{2}(\Gamma)$ is continuous and compact and 
using Aubin compactness theorem, we can extract subsequences of $\left\{v_{m}(t)\right\}$ and $\left\{u_{m}(t)\right\}$ such that

$$
\begin{array}{llll}
u_{m}(t) \longrightarrow u(t) & \text { a.e. in } Q, & u_{m}^{\prime}(t) \longrightarrow u^{\prime}(t) & \text { a.e. in } Q, \\
v_{m}(t) \longrightarrow v(t) & \text { a.e. in } Q, & v_{m}^{\prime}(t) \longrightarrow v^{\prime}(t) & \text { a.e. in } Q, \\
v_{m}(t) \longrightarrow v(t) & \text { a.e. on } \Sigma, & v_{m}^{\prime}(t) \longrightarrow v^{\prime}(t) & \text { a.e. on } \Sigma,
\end{array}
$$

where we denote $Q=\Omega \times(0, T]$ and $\Sigma=\Gamma \times(0, T]$.

On the other hand, from the first, the second estimate, and assumptions we have

$$
\begin{gathered}
\left\{g_{1}\left(u_{m}^{\prime}\right)\right\}, \quad\left\{g_{2}\left(v_{m}^{\prime}\right)\right\} \text { are bounded in } L^{2}(Q), \\
\left\{h_{1} * J_{1}\left(\Delta u_{m}\right)\right\} \text { is bounded in } L^{2}(Q), \\
\left\{h_{2} * J_{2}\left(v_{m}\right)\right\} \text { is bounded in } L^{2}(\Sigma), \\
\left\{g\left(v_{m}^{\prime}\right)\right\} \text { is bounded in } L^{2}(\Sigma) .
\end{gathered}
$$

Hence, combining (3.33)-(3.37) and Lions' lemma we deduce that

$$
\begin{array}{rr}
g_{1}\left(u_{m}^{\prime}\right) \longrightarrow g_{1}\left(u^{\prime}\right) & \text { weakly in } L^{2}(Q), \\
g_{2}\left(v_{m}^{\prime}\right) \longrightarrow g_{2}\left(v^{\prime}\right) & \text { weakly in } L^{2}(Q), \\
h_{2} * J_{2}\left(v_{m}\right) \longrightarrow h_{2} * J_{2}(v) & \text { weakly in } L^{2}(\Sigma), \\
g\left(v_{m}^{\prime}\right) \longrightarrow g\left(v^{\prime}\right) & \text { weakly in } L^{2}(\Sigma),
\end{array}
$$

Also

$$
h_{1} * J_{1}\left(\Delta u_{m}\right) \longrightarrow \chi \quad \text { weakly in } L^{2}(Q)
$$

where $\chi \in L^{2}(Q)$. Next we will show that $\chi=h_{1} * J_{1}(\Delta u)$.

From (3.27), (3.29), and (3.32), we have

$$
\begin{gathered}
u_{m}^{\prime \prime} \longrightarrow u^{\prime \prime} \quad \text { weakly in } L^{2}(Q), \\
v_{m}^{\prime \prime} \longrightarrow v^{\prime \prime} \quad \text { weakly in } L^{2}(Q), \\
\Delta u_{m} \longrightarrow \Delta u \quad \text { weakly in } L^{2}(Q) .
\end{gathered}
$$

Moreover from the preceding estimates, we deduce that

$$
\begin{gathered}
u_{m} \longrightarrow u \quad \text { strongly in } C^{0}\left(0, T ; H_{0}^{1}(\Omega)\right), \\
v_{m} \longrightarrow v \quad \text { strongly in } C^{0}(0, T ; V) .
\end{gathered}
$$

Hence we can pass to the limit in (3.3) to obtain

$$
u^{\prime \prime}+\beta_{1} \Delta^{2} u-\left(1+\|\nabla u\|^{2}+\|\nabla v\|^{2}\right) \Delta u-\beta_{2} \Delta u^{\prime}+a(u-v)+g_{1}\left(u^{\prime}\right)=\chi \quad \text { in } D^{\prime}(Q) .
$$


But consider that $u^{\prime \prime}, \chi,\left(1+\|\nabla u\|^{2}+\|\nabla v\|^{2}\right) \Delta u, \Delta u^{\prime}, a(u-v), g_{1}\left(u^{\prime}\right) \in L^{2}(Q)$, we deduce that $\Delta^{2} u \in L^{2}(Q)$, and moreover

$u^{\prime \prime}+\beta_{1} \Delta^{2} u-\left(1+\|\nabla u\|^{2}+\|\nabla v\|^{2}\right) \Delta u-\beta_{2} \Delta u^{\prime}+a(u-v)+g_{1}\left(u^{\prime}\right)=\chi \quad$ in $L^{2}\left(0, T ; L^{2}(\Omega)\right)$.

Further, we can easily verify that (1.3) holds in the sense of $L^{2}\left(0, T ; L^{2}(\partial \Omega)\right)$, that is,

$$
u=\frac{\partial u}{\partial \nu}=0 \quad \text { in } L^{2}\left(0, T ; L^{2}(\partial \Omega)\right)
$$

Now considering $w=u_{m}$ in (3.3) and integrating it over $[0, \mathrm{~T}]$ we obtain

$$
\begin{aligned}
\int_{0}^{T}\left(u_{m}^{\prime \prime}(t), u_{m}(t)\right) d t+\int_{0}^{T}\left(\beta_{1} \Delta u_{m}(t), \Delta u_{m}(t)\right) d t \\
\quad+\int_{0}^{T}\left(1+\left\|\nabla u_{m}\right\|^{2}+\left\|\nabla v_{m}\right\|^{2}\right)\left(\nabla u_{m}(t), \nabla u_{m}(t)\right) d t \\
\quad+\int_{0}^{T} \beta_{2}\left(\nabla u_{m}^{\prime}(t), \nabla u_{m}(t)\right) d t+\int_{0}^{T}\left(a u_{m}(t), u_{m}(t)\right) d t \\
\quad-\int_{0}^{T}\left(a v_{m}(t), u_{m}(t)\right) d t+\int_{0}^{T}\left(g_{1}\left(u_{m}^{\prime}(t)\right), u_{m}(t)\right) d t \\
=\int_{0}^{T} \int_{0}^{t} h_{1}(t-s)\left(J_{1}\left(\Delta u_{m}(s)\right), u_{m}(t)\right) d s d t
\end{aligned}
$$

Further, from the first and second estimates and using Aubin-lions theorem we infer

$$
u_{m} \longrightarrow u \quad \text { strongly in } L^{2}\left(0, T ; L^{2}(\Omega)\right)
$$

Thus, using the convergences (3.39), (3.40), and (3.42), we can pass to the limit in (3.47) and obtain

$$
\begin{aligned}
\lim _{m \rightarrow \infty} \int_{0}^{T} & \beta_{1}\left\|\Delta u_{m}(t)\right\|^{2} d t \\
= & -\int_{0}^{T}\left(u^{\prime \prime}(t), u(t)\right) d t-\int_{0}^{T}\left(\beta_{2} \nabla u^{\prime}(t), \nabla u(t)\right) d t-\int_{0}^{T}(a u(t), u(t)) d t \\
& +\int_{0}^{T}(a v(t), u(t)) d t+\int_{0}^{T}\left(1+\|\nabla u(t)\|^{2}+\|\nabla v(t)\|^{2}\right)(\nabla u(t), \nabla u(t)) d t \\
& -\int_{0}^{T}\left(g_{1}\left(u^{\prime}(t)\right), u(t)\right) d t+\int_{0}^{T}(\chi, u(t)) d t .
\end{aligned}
$$


14 Existence and uniform decay of solutions

Hence, combining (3.45) and (3.46) into (3.49) and using generalized Green formula, we obtain

$$
\lim _{m \rightarrow \infty} \int_{0}^{T}\left\|\Delta u_{m}(t)\right\|^{2} d t=\int_{0}^{T}\|\Delta u(t)\|^{2} d t
$$

So, from (3.42) and (3.50) we infer that

$$
\Delta u_{m} \longrightarrow \Delta u \quad \text { strongly in } L^{2}\left(0, T ; L^{2}(\Omega)\right)
$$

Therefore,

$$
\Delta u_{m} \rightarrow \Delta u \quad \text { a.e. in } \Omega,
$$

and consequently

$$
J_{1}\left(\Delta u_{m}\right) \longrightarrow J_{1}(\Delta u) \quad \text { a.e. in } \Omega \text {. }
$$

Combining (3.35) and (3.53), we obtain

$$
h_{1} * J_{1}\left(\Delta u_{m}\right) \longrightarrow \chi=h_{1} * J_{1}(\Delta u) \quad \text { weakly in } L^{2}\left(0, T ; L^{2}(\Omega)\right) .
$$

Therefore, the above convergences are sufficient to pass to the limit in problem (1.1)(1.7).

\section{Uniqueness of the solution}

Let $\left(u_{1}, v_{1}\right)$ and $\left(u_{2}, v_{2}\right)$ be two solutions of couple system $(1.1)-(1.7)$, then $(u, v):=\left(u_{1}-\right.$ $\left.u_{2}, v_{1}-v_{2}\right)$ verifies

$$
\begin{aligned}
\left(u^{\prime \prime}, w\right)+ & \beta_{1}(\Delta u, \Delta w) \\
& +\left(\left[\left(\left\|\nabla u_{1}\right\|^{2}+\left\|\nabla v_{1}\right\|^{2}\right) \nabla u_{1}-\left(\left\|\nabla u_{2}\right\|^{2}+\left\|\nabla v_{2}\right\|^{2}\right) \nabla u_{2}\right], \nabla w\right) \\
& +(\nabla u, \nabla w)+\beta_{2}\left(\nabla u^{\prime}, \nabla w\right)+(a u, w)-(a v, w)+\left(\left[g_{1}\left(u_{1}^{\prime}\right)-g_{1}\left(u_{2}^{\prime}\right)\right], w\right) \\
= & \int_{0}^{t} h_{1}(t-s)\left(\left[J_{1}\left(\Delta u_{1}(s)\right)-J_{1}\left(\Delta u_{2}(s)\right)\right], w(t)\right) d s, \quad w \in H_{0}^{2}(\Omega), \\
\left(v^{\prime \prime}, w\right)+ & \left(\left[\left(\left\|\nabla u_{1}\right\|^{2}+\left\|\nabla v_{1}\right\|^{2}\right) \nabla v_{1}-\left(\left\|\nabla u_{2}\right\|^{2}+\left\|\nabla v_{2}\right\|^{2}\right) \nabla v_{2}\right], \nabla w\right) \\
& +(\nabla v, \nabla w)+\beta_{3}\left(\nabla v^{\prime}, \nabla w\right)+(a v, w)-(a u, w)+\left(\left[g_{2}\left(v_{1}^{\prime}\right)-g_{2}\left(v_{2}^{\prime}\right)\right], w\right) \\
& +(v, w)_{\Gamma}+\left(v^{\prime}, w\right)_{\Gamma}+\left(\left[g\left(v_{1}^{\prime}\right)-g\left(v_{2}^{\prime}\right)\right], w\right)_{\Gamma} \\
= & \int_{0}^{t} h_{2}(t-s)\left(\left[J_{2}\left(v_{1}(s)\right)-J_{2}\left(v_{2}(s)\right)\right], w(t)\right)_{\Gamma} d s, \quad w \in V .
\end{aligned}
$$


Substituting $w=u^{\prime}(t)$ in (4.1) and $w=v^{\prime}(t)$ in (4.2), respectively, meanwhile observing that $g, g_{i}, i=1,2$, are nondecreasing and using the first estimate, we get

$$
\begin{aligned}
\frac{d}{d t}\left\{\frac{1}{2}\left\|u^{\prime}(t)\right\|^{2}+\frac{\beta_{1}}{2}\|\Delta u(t)\|^{2}+\frac{1}{2}\|\nabla u(t)\|^{2}+\frac{a}{2}\|u(t)\|^{2}\right\} \\
\quad-\left(a v(t), u^{\prime}(t)\right)+\beta_{2}\left\|\nabla u^{\prime}(t)\right\|^{2} \\
\leq C_{27}\|\nabla u\|\left\|\nabla u^{\prime}\right\|+\int_{0}^{t} h_{1}(t-s)\left(\left[J_{1}\left(\Delta u_{1}(s)\right)-J_{1}\left(\Delta u_{2}(s)\right)\right], u^{\prime}(t)\right) d s \\
\leq \frac{C_{27}^{2}}{4 \eta}\|\nabla u\|^{2}+\eta\left\|\nabla u^{\prime}(t)\right\|^{2}+\frac{C_{28}}{2}\left\|u^{\prime}(t)\right\|^{2} \\
+\frac{1}{2} \int_{0}^{t}\left|h_{1}(t-s)\right|^{2}\left\|J_{1}\left(\Delta u_{1}(s)\right)-J_{1}\left(\Delta u_{2}(s)\right)\right\|^{2} d s
\end{aligned}
$$

$$
\begin{aligned}
\frac{d}{d t}\left\{\frac{1}{2}\left\|v^{\prime}(t)\right\|^{2}+\frac{1}{2}\|\nabla v(t)\|^{2}+\frac{a}{2}\|v(t)\|^{2}+\frac{1}{2}\|v(t)\|_{\Gamma}^{2}\right\} \\
-\left(a u(t), v^{\prime}(t)\right)+\beta_{3}\left\|\nabla v^{\prime}(t)\right\|^{2}+\left\|v^{\prime}(t)\right\|_{\Gamma}^{2} \\
\leq C_{29}\|\nabla v\|\left\|\nabla v^{\prime}\right\|+\int_{0}^{t} h_{2}(t-s)\left(\left[J_{2}\left(v_{1}(s)\right)-J_{2}\left(v_{2}(s)\right)\right], v^{\prime}(t)\right)_{\Gamma} d s \\
\leq \frac{C_{29}^{2}}{4 \eta}\|\nabla v\|^{2}+\eta\left\|\nabla v^{\prime}(t)\right\|^{2}+\eta C_{30}\left\|v^{\prime}(t)\right\|_{\Gamma}^{2} \\
\quad+\frac{1}{4 \eta} \int_{0}^{t}\left|h_{2}(t-s)\right|^{2}\left\|J_{2}\left(v_{1}(s)\right)-J_{2}\left(v_{2}(s)\right)\right\|_{\Gamma}^{2} d s,
\end{aligned}
$$

where $\eta>0$ is arbitrary.

Furthermore, noticing $J_{1}$ global Lipschitz and Assumption (A.5), we have

$$
\begin{aligned}
& \int_{0}^{t}\left|h_{1}(t-s)\right|^{2}\left\|J_{1}\left(\Delta u_{1}(s)\right)-J_{1}\left(\Delta u_{2}(s)\right)\right\|^{2} d s \\
& \quad \leq C_{31}\left\|h_{1}\right\|_{L^{1}(0, \infty)}^{2} \int_{0}^{t}\|\Delta u(s)\|^{2} d s \leq C_{32} \int_{0}^{t}\|\Delta u(s)\|^{2} d s, \\
& \int_{0}^{t}\left|h_{2}(t-s)\right|^{2}\left\|J_{2}\left(v_{1}(s)\right)-J_{2}\left(v_{2}(s)\right)\right\|_{\Gamma}^{2} d s \\
& \quad \leq C_{33} \int_{0}^{t}\left|h_{2}(t-s)\right|\|\nabla v(s)\|^{2} d s \leq C_{34} \int_{0}^{t}\|\nabla v(s)\|^{2} d s .
\end{aligned}
$$


Hence, integrating (4.3) and (4.4) over $(0, t)$, using (4.5), then adding the results together we obtain

$$
\begin{aligned}
\frac{1}{2} \| u^{\prime}(t) & \left\|^{2}+\frac{\beta_{1}}{2}\right\| \Delta u(t)\left\|^{2}+\frac{1}{2}\right\| \nabla u(t)\left\|^{2}+\frac{a}{2}\right\| u(t)\left\|^{2}+\left(\beta_{2}-\eta\right) \int_{0}^{t}\right\| \nabla u^{\prime}(s) \|^{2} d s \\
& +\frac{1}{2}\left\|v^{\prime}(t)\right\|^{2}+\frac{1}{2}\|\nabla v(t)\|^{2}+\frac{a}{2}\|v(t)\|^{2}+\frac{1}{2}\|v(t)\|_{\Gamma}^{2}-\int_{\Omega} a u(t) v(t) d x \\
& +\left(\beta_{3}-\eta\right) \int_{0}^{t}\left\|\nabla v^{\prime}(s)\right\|^{2} d s+\left(1-\eta C_{30}\right) \int_{0}^{t}\left\|v^{\prime}(s)\right\|_{\Gamma}^{2} d s \\
\leq & \frac{C_{27}^{2}}{4 \eta} \int_{0}^{t}\|\nabla u(s)\|^{2} d s+\frac{C_{28}}{2} \int_{0}^{t}\left\|u^{\prime}(s)\right\|^{2} d s+\frac{C_{35}}{2} \int_{0}^{t}\|\Delta u(s)\|^{2} d s \\
& +\frac{C_{29}^{2}}{4 \eta} \int_{0}^{t}\|\nabla v(s)\|^{2} d s+\frac{C_{36}}{4 \eta} \int_{0}^{t}\|\nabla v(s)\|^{2} d s,
\end{aligned}
$$

for $\eta>0$ small enough.

Meanwhile, from (3.31) and (3.32), we can deduce that $\left\|v^{\prime}(t)\right\|_{\Gamma}^{2}$ is continuous on $[0, T]$, see [14].

Thus, combining the last inequality and using Gronwall's lemma, we obtain that

$$
\begin{gathered}
\left\|u^{\prime}(t)\right\|=\|\Delta u(t)\|=0, \\
\left\|v^{\prime}(t)\right\|=\|\nabla v(t)\|=\|v(t)\|_{\Gamma}=\left\|v^{\prime}(t)\right\|_{\Gamma}=0 .
\end{gathered}
$$

\section{Asymptotic behavior of the solution}

In this section, we follow the additional assumptions that appeared in Theorem 2.1. According to the definition of $E(t)$, the derivative of the energy is

$$
\begin{aligned}
E^{\prime}(t)= & -\left(g_{1}\left(u^{\prime}(t)\right), u^{\prime}(t)\right)-\left(g_{2}\left(v^{\prime}(t)\right), v^{\prime}(t)\right)-\left(g\left(v^{\prime}(t)\right), v^{\prime}(t)\right)_{\Gamma} \\
& -\beta_{2}\left\|\nabla u_{m}^{\prime}\right\|^{2}-\beta_{3}\left\|\nabla v_{m}^{\prime}\right\|^{2}-\left\|v^{\prime}(t)\right\|_{\Gamma}^{2} \\
& +\int_{0}^{t} h_{1}(t-s)\left(J_{1}(\Delta u(s)), u^{\prime}(t)\right) d s+\int_{0}^{t} h_{2}(t-s)\left(J_{2}(v(s)), v^{\prime}(t)\right)_{\Gamma} d s .
\end{aligned}
$$

Define

$$
\begin{aligned}
& \left(h_{1} \square u\right)(t):=\int_{0}^{t} h_{1}(t-s)\left\|J_{1}(\Delta u(s))-u(t)\right\|^{2} d s, \\
& \left(h_{2} \square v\right)(t):=\int_{0}^{t} h_{2}(t-s)\left\|J_{2}(v(s))-v(t)\right\|_{\Gamma}^{2} d s .
\end{aligned}
$$


Hence we have

$$
\begin{aligned}
& \int_{0}^{t} h_{1}(t-s)\left(J_{1}(\Delta u(s)), u^{\prime}(t)\right) d s \\
& \quad=-\frac{1}{2}\left(h_{1} \square u\right)^{\prime}(t)+\frac{1}{2}\left(h_{1}^{\prime} \square u\right)(t)+\frac{1}{2} \frac{d}{d t}\left\{\left(\int_{0}^{t} h_{1}(s) d s\right)\|u(t)\|^{2}\right\}-\frac{1}{2} h_{1}(t)\|u(t)\|^{2}, \\
& \int_{0}^{t} h_{2}(t-s)\left(J_{2}(v(s)), v^{\prime}(t)\right)_{\Gamma} d s \\
& \quad=-\frac{1}{2}\left(h_{2} \square v\right)^{\prime}(t)+\frac{1}{2}\left(h_{2}^{\prime} \square v\right)(t)+\frac{1}{2} \frac{d}{d t}\left\{\left(\int_{0}^{t} h_{2}(s) d s\right)\|v(t)\|_{\Gamma}^{2}\right\}-\frac{1}{2} h_{2}(t)\|v(t)\|_{\Gamma}^{2} .
\end{aligned}
$$

Define the modified energy

$$
\begin{aligned}
e(t)= & \frac{1}{2}\left\|u^{\prime}(t)\right\|^{2}+\frac{\beta_{1}}{2}\|\Delta u(t)\|^{2}+\frac{1}{2}\left\|v^{\prime}(t)\right\|^{2}+\frac{1}{2}\|\nabla v(t)\|^{2}+\frac{1}{2}\|\nabla u(t)\|^{2} \\
& +\frac{1}{4}\left(\|\nabla u(t)\|^{2}+\|\nabla v(t)\|^{2}\right)^{2}+\frac{1}{2}\left(a-\int_{0}^{t} h_{1}(s) d s\right)\|u(t)\|^{2}+\frac{a}{2}\|v(t)\|^{2} \\
& -\int_{\Omega} a u(t) v(t) d x+\frac{1}{2}\left(1-\int_{0}^{t} h_{2}(s) d s\right)\|v(t)\|_{\Gamma}^{2} \\
& +\frac{1}{2}\left(h_{1} \square u\right)(t)+\frac{1}{2}\left(h_{2} \square v\right)(t)+\frac{1}{\gamma+2} h_{2}(t)\|v(t)\|_{\gamma+2, \Gamma \cdot}^{\gamma+2}
\end{aligned}
$$

Consider Assumption (A.7), it is easy to know that $e(t)>0$. Moreover,

$$
\begin{aligned}
e^{\prime}(t)= & -\left(g_{1}\left(u^{\prime}(t)\right), u^{\prime}(t)\right)-\left(g_{2}\left(v^{\prime}(t)\right), v^{\prime}(t)\right)-\beta_{2}\left\|\nabla u^{\prime}(t)\right\|^{2} \\
& -\beta_{3}\left\|\nabla v^{\prime}(t)\right\|^{2}-\left(g\left(v^{\prime}(t)\right), v^{\prime}(t)\right)_{\Gamma}+\frac{1}{\gamma+2} h_{2}^{\prime}(t)\|v(t)\|_{\gamma+2, \Gamma}^{\gamma+2} \\
& +h_{2}(t)\left(|v(t)|^{\gamma} v(t), v^{\prime}(t)\right)_{\Gamma}-\frac{1}{2} h_{1}(t)\|u(t)\|^{2}-\frac{1}{2} h_{2}(t)\|v(t)\|_{\Gamma}^{2} \\
& +\frac{1}{2}\left(h_{1}^{\prime} \square u\right)(t)+\frac{1}{2}\left(h_{2}^{\prime} \square v\right)(t)-\left\|v^{\prime}(t)\right\|_{\Gamma}^{2} .
\end{aligned}
$$

Using Young's inequality, we know that

$$
h_{2}(t)\left(|v(t)|^{\gamma} v(t), v^{\prime}(t)\right)_{\Gamma} \leq \eta h_{2}(t)\left\|v^{\prime}(t)\right\|_{\gamma+2, \Gamma}^{\gamma+2}+\eta^{-1 /(\gamma+1)} h_{2}(t)\|v(t)\|_{\gamma+2, \Gamma}^{\gamma+2},
$$


where $\eta>0$ is arbitrary. Hence, by Assumptions (A.3) and (A.6) for $\gamma=\rho$, we have for all $t \geq t_{0}$ that

$$
\begin{aligned}
e^{\prime}(t) \leq & -\left(g_{1}\left(u^{\prime}(t)\right), u^{\prime}(t)\right)-\left(g_{2}\left(v^{\prime}(t)\right), v^{\prime}(t)\right)-\beta_{2}\left\|\nabla u^{\prime}(t)\right\|^{2} \\
& -\beta_{3}\left\|\nabla v^{\prime}(t)\right\|^{2}-\left(\alpha_{3}-\eta h_{2}(t)\right)\left\|v^{\prime}(t)\right\|_{\rho+2, \Gamma}^{\rho+2} \\
& -\left(\frac{M_{3}}{\gamma+2}-\eta^{-1 /(\gamma+1)}\right) h_{2}(t)\|v(t)\|_{\gamma+2, \Gamma}^{\gamma+2}-\frac{1}{2} h_{1}(t)\|u(t)\|^{2} \\
& -\frac{1}{2} h_{2}(t)\|v(t)\|_{\Gamma}^{2}-\frac{M_{3}}{2}\left(h_{1} \square u\right)(t)-\frac{M_{3}}{2}\left(h_{2} \square v\right)(t)-\left\|v^{\prime}(t)\right\|_{\Gamma}^{2}
\end{aligned}
$$

Choosing $\eta=\alpha_{3} / 2\left\|h_{2}\right\|_{\infty}$ (then $\left.\alpha_{3}-\eta \geq \alpha_{3} / 2\right)$, we deduce

$$
\begin{aligned}
e^{\prime}(t) \leq & -\left(g_{1}\left(u^{\prime}(t)\right), u^{\prime}(t)\right)-\left(g_{2}\left(v^{\prime}(t)\right), v^{\prime}(t)\right) \\
& -\beta_{2}\left\|\nabla u^{\prime}(t)\right\|^{2}-\beta_{3}\left\|\nabla v^{\prime}(t)\right\|^{2}-\frac{\alpha_{3}}{2}\left\|v^{\prime}(t)\right\|_{\rho+2, \Gamma}^{\rho+2} \\
& -\xi_{1} h_{2}(t)\|v(t)\|_{\gamma+2, \Gamma}^{\gamma+2}-\frac{1}{2} h_{1}(t)\|u(t)\|^{2}-\frac{1}{2} h_{2}(t)\|v(t)\|_{\Gamma}^{2} \\
& -\frac{M_{3}}{2}\left(h_{1} \square u\right)(t)-\frac{M_{3}}{2}\left(h_{2} \square v\right)(t)-\left\|v^{\prime}(t)\right\|_{\Gamma}^{2},
\end{aligned}
$$

where $\xi_{1}=M_{3} /(\gamma+2)-\left(\alpha_{3} / 2\left\|h_{2}\right\|_{\infty}\right)^{-1 /(\gamma+1)}>0$. Furthermore, from Assumption (A.7), we have

$$
\begin{aligned}
E(t) \leq & \frac{1}{2}\left(\left\|u^{\prime}(t)\right\|^{2}+\left\|v^{\prime}(t)\right\|^{2}+\|\nabla u(t)\|^{2}+\|\nabla v(t)\|^{2}\right)+\frac{a}{2}\|v(t)\|^{2} \\
& +\frac{\beta_{1}}{2}\|\Delta u(t)\|^{2}+\frac{1}{4}\left(\|\nabla u(t)\|^{2}+\|\nabla v(t)\|^{2}\right)^{2}-\int_{\Omega} a u(t) v(t) d x \\
& +\frac{1}{2 l_{1}}\left(a-\int_{0}^{t} h_{1}(s) d s\right)\|u(t)\|^{2}+\frac{1}{2 l_{2}}\left(1-\int_{0}^{t} h_{2}(s) d s\right)\|v(t)\|_{\Gamma}^{2} \\
\leq & \min \left\{l_{1}, l_{2}\right\}^{-1} e(t) .
\end{aligned}
$$

For every $\varepsilon>0$, we define the perturbed energy by setting

$$
e_{\varepsilon}(t)=e(t)+\varepsilon \psi(t), \quad \psi(t)=\left(u^{\prime}(t), u(t)\right)+\left(v^{\prime}(t), v(t)\right) .
$$

Lemma 5.1. There exists $\xi_{2}>0$ such that

$$
\left|e_{\varepsilon}(t)-e(t)\right| \leq \varepsilon \xi_{2} e(t) \quad \forall t \geq 0 .
$$


Proof. From (2.2) and (5.10), we obtain

$$
\begin{aligned}
|\psi(t)| & \leq \sqrt{\mu_{2}}\left\|u^{\prime}(t)\right\|\|\nabla u(t)\|+\sqrt{\mu_{2}}\left\|v^{\prime}(t)\right\|\|\nabla v(t)\| \\
& \leq \sqrt{\mu_{2}}\left(\frac{1}{2}\left\|u^{\prime}(t)\right\|^{2}+\frac{1}{2}\|\nabla u(t)\|^{2}+\frac{1}{2}\left\|v^{\prime}(t)\right\|^{2}+\frac{1}{2}\|\nabla v(t)\|^{2}\right) \\
& \leq \sqrt{\mu_{2}} e(t) .
\end{aligned}
$$

Hence we have

$$
\left|e_{\varepsilon}(t)-e(t)\right| \leq \varepsilon \xi_{2} e(t) \quad \forall t \geq 0,
$$

where $\xi_{2}=\sqrt{\mu_{2}}$.

Lemma 5.2. There exist $\xi_{3}>0$ and $\bar{\varepsilon}$ such that for $\varepsilon \in(0, \bar{\varepsilon}]$

$$
e_{\varepsilon}^{\prime}(t) \leq-\varepsilon \xi_{3} e(t)
$$

Proof. By using the problem (1.1)-(1.7), we obtain

$$
\begin{aligned}
\psi^{\prime}(t)= & \left\|u^{\prime}(t)\right\|^{2}+\left\|v^{\prime}(t)\right\|^{2}-\beta_{1}\|\Delta u(t)\|^{2}-a\|u(t)\|^{2}-a\|v(t)\|^{2}+2 \int_{\Omega} a u(t) v(t) d x \\
& -\|\nabla u\|^{2}-\|\nabla v\|^{2}-\left(\|\nabla u\|^{2}+\|\nabla v\|^{2}\right)^{2}-\|v(t)\|_{\Gamma}^{2}-\beta_{2}\left(\nabla u^{\prime}, \nabla u\right)-\beta_{3}\left(\nabla v^{\prime}, \nabla v\right) \\
& -\left(v^{\prime}(t), v(t)\right)_{\Gamma}-\left(g\left(v^{\prime}(t)\right), v(t)\right)_{\Gamma}-\left(g_{1}\left(u^{\prime}(t)\right), u(t)\right)-\left(g_{2}\left(v^{\prime}(t)\right), v(t)\right) \\
& +\int_{0}^{t} h_{1}(t-s)\left(J_{1}(\Delta u(s)), u(t)\right) d s+\int_{0}^{t} h_{2}(t-s)\left(J_{2}(v(s)), v(t)\right)_{\Gamma} d s \\
= & -e(t)+\frac{3}{2}\left\|u^{\prime}(t)\right\|^{2}+\frac{3}{2}\left\|v^{\prime}(t)\right\|^{2}-\frac{\beta_{1}}{2}\|\Delta u(t)\|^{2}-\frac{1}{2}\|\nabla u(t)\|^{2}-\frac{1}{2}\|\nabla v(t)\|^{2} \\
& -\frac{3}{4}\left(\|\nabla u(t)\|^{2}+\|\nabla v(t)\|^{2}\right)^{2}-\beta_{2}\left(\nabla u^{\prime}, \nabla u\right)-\beta_{3}\left(\nabla v^{\prime}, \nabla v\right)+\int_{\Omega} a u(t) v(t) d x \\
& -\frac{a}{2}\|v(t)\|^{2}-\frac{a}{2}\|u(t)\|^{2}-\frac{1}{2} \int_{0}^{t} h_{1}(s) d s\|u(t)\|^{2}-\frac{1}{2}\|v(t)\|_{\Gamma}^{2}-\frac{1}{2} \int_{0}^{t} h_{2}(s) d s\|v(t)\|_{\Gamma}^{2} \\
& +\frac{1}{2}\left(h_{1} \square u\right)(t)+\frac{1}{2}\left(h_{2} \square v\right)(t)-\left(v^{\prime}(t), v(t)\right)_{\Gamma}-\left(g\left(v^{\prime}(t)\right), v(t)\right)_{\Gamma} \\
& -\left(g_{1}\left(u^{\prime}(t)\right), u(t)\right)-\left(g_{2}\left(v^{\prime}(t)\right), v(t)\right)+\int_{0}^{t} h_{1}(t-s)\left(J_{1}(\Delta u(s)), u(t)\right) d s \\
& +\int_{0}^{t} h_{2}(t-s)\left(J_{2}(v(s)), v(t)\right)_{\Gamma} d s+\frac{1}{\gamma+2} h_{2}(t)\|v(t)\|_{\gamma+2, \Gamma}^{\gamma+2} .
\end{aligned}
$$


Note that

$$
\begin{aligned}
& \int_{0}^{t} h_{1}(t-s)\left(J_{1}(\Delta u(s)), u(t)\right) d s \\
& \quad \int_{0}^{t} h_{1}(t-s)\left(J_{1}(\Delta u(s))-u(t), u(t)\right) d s+\int_{0}^{t} h_{1}(t-s)\|u(t)\|^{2} d s \\
& \leq \frac{1}{2} \int_{0}^{t} h_{1}(t-s)\left\|J_{1}(\Delta u(s))-u(t)\right\|^{2} d s+\frac{3}{2}\|u(t)\|^{2} \int_{0}^{t} h_{1}(s) d s \\
&= \frac{1}{2}\left(h_{1} \square u\right)(t)+\frac{3}{2}\|u(t)\|^{2} \int_{0}^{t} h_{1}(s) d s, \\
& \int_{0}^{t} h_{2}(t-s)\left(J_{2}(v(s)), v(t)\right)_{\Gamma} d s \\
&=\int_{0}^{t} h_{2}(t-s)\left(J_{2}(v(s))-v(t), v(t)\right)_{\Gamma} d s+\int_{0}^{t} h_{2}(t-s)\|v(t)\|_{\Gamma}^{2} d s \\
& \leq \frac{1}{2} \int_{0}^{t} h_{2}(t-s)\left\|J_{2}(v(s))-v(t)\right\|_{\Gamma}^{2} d s+\frac{3}{2}\|v(t)\|_{\Gamma}^{2} \int_{0}^{t} h_{2}(s) d s \\
&=\frac{1}{2}\left(h_{2} \square v\right)(t)+\frac{3}{2}\|v(t)\|_{\Gamma}^{2} \int_{0}^{t} h_{2}(s) d s .
\end{aligned}
$$

Using Sobolev embedding relation (2.2), we obtain

$$
\left|\left(v(t), v^{\prime}(t)\right)_{\Gamma}\right| \leq \mu_{3}|| \nabla v(t)\left|\left\||| v^{\prime}(t)\right\|_{\Gamma} \leq \eta\|\nabla v(t)\|^{2}+\frac{\mu_{3}^{2}}{4 \eta}\left\|v^{\prime}(t)\right\|_{\Gamma}^{2} .\right.
$$

Also by Assumption (A.3), Hölder's inequality, and Young's inequality we deduce that

$$
\begin{aligned}
\left|\left(g\left(v^{\prime}(t)\right), v(t)\right)_{\Gamma}\right| & \leq \alpha_{4}\left\|v^{\prime}(t)\right\|_{\rho+2, \Gamma}^{\rho+1}\|v(t)\|_{\rho+2, \Gamma} \\
& \leq \theta(\eta)\left\|v^{\prime}(t)\right\|_{\rho+2, \Gamma}^{\rho+2}+\eta\|v(t)\|_{\rho+2, \Gamma}^{\rho+2}, \\
\left|\int_{\Omega} a u(t) v(t) d x\right| & \leq a \sqrt{\mu_{1} \mu_{2}}\|\Delta u\|\|\nabla v\| \\
& \leq a \frac{\sqrt{\mu_{1} \mu_{2}}}{2}\left(\|\Delta u\|^{2}+\|\nabla v\|^{2}\right) .
\end{aligned}
$$

Moreover

$$
\begin{aligned}
& \beta_{2}\left(\nabla u^{\prime}(t), \nabla u(t)\right) \leq \beta_{2} \eta\|\nabla u(t)\|^{2}+\frac{\beta_{2}}{4 \eta}\left\|\nabla u^{\prime}(t)\right\|^{2}, \\
& \beta_{3}\left(\nabla v^{\prime}(t), \nabla v(t)\right) \leq \beta_{3} \eta\|\nabla v(t)\|^{2}+\frac{\beta_{3}}{4 \eta}\left\|\nabla v^{\prime}(t)\right\|^{2} .
\end{aligned}
$$


From Assumption (A.2) and (5.8), we obtain

$$
\begin{aligned}
& \left|-\left(g_{1}\left(u^{\prime}(t)\right), u(t)\right)-\left(g_{2}\left(v^{\prime}(t)\right), v(t)\right)\right| \\
& \leq \frac{\eta}{\mu_{1}}\|u(t)\|^{2}+\frac{\eta}{\mu_{2}}\|v(t)\|^{2}+\frac{\mu_{1}}{4 \eta} \int_{\Omega} g_{1}\left(u^{\prime}(t)\right)^{2} d x+\frac{\mu_{2}}{4 \eta} \int_{\Omega} g_{2}\left(v^{\prime}(t)\right)^{2} d x \\
& \leq \eta\left(\|\Delta u(t)\|^{2}+\|\nabla v(t)\|^{2}\right)+\frac{\left(\mu_{1}+\mu_{2}\right) \alpha_{2}}{4 \eta} \int_{\Omega}\left\{u^{\prime}(t) g_{1}\left(u^{\prime}(t)\right)+v^{\prime}(t) g_{2}\left(v^{\prime}(t)\right)\right\} d x \\
& \leq \eta\left(\|\Delta u(t)\|^{2}+\|\nabla v(t)\|^{2}\right)+\frac{\left(\mu_{1}+\mu_{2}\right) \alpha_{2}}{4 \eta} \\
& \times\left[-e^{\prime}(t)-\beta_{2}\left\|\nabla u^{\prime}(t)\right\|^{2}-\beta_{3}\left\|\nabla v^{\prime}(t)\right\|^{2}-\frac{\alpha_{3}}{2}\left\|v^{\prime}(t)\right\|_{\rho+2, \Gamma}^{\rho+2}\right. \\
& -\xi_{1} h_{2}(t)\|v(t)\|_{\gamma+2, \Gamma}^{\gamma+2}-\frac{1}{2} h_{1}(t)\|u(t)\|^{2}-\frac{1}{2} h_{2}(t)\|v(t)\|_{\Gamma}^{2} \\
& \left.-\frac{M_{3}}{2}\left(h_{1} \square u\right)(t)-\frac{M_{3}}{2}\left(h_{2} \square v\right)(t)-\left\|v^{\prime}(t)\right\|_{\Gamma}^{2}\right] \text {, } \\
& \frac{3}{2} \int_{\Omega}\left\{\left|u^{\prime}(t)\right|^{2}+\left|v^{\prime}(t)\right|^{2}\right\} d x \\
& \leq \frac{3}{2 \alpha_{1}} \int_{\Omega}\left\{u^{\prime}(t) g_{1}\left(u^{\prime}(t)\right)+v^{\prime}(t) g_{2}\left(v^{\prime}(t)\right)\right\} d x \\
& \leq \frac{3}{2 \alpha_{1}}\left[-e^{\prime}(t)-\beta_{2}\left\|\nabla u^{\prime}(t)\right\|^{2}-\beta_{3}\left\|\nabla v^{\prime}(t)\right\|^{2}-\frac{\alpha_{3}}{2}\left\|v^{\prime}(t)\right\|_{\rho+2, \Gamma}^{\rho+2}\right. \\
& -\xi_{1} h_{2}(t)\|v(t)\|_{\gamma+2, \Gamma}^{\gamma+2}-\frac{1}{2} h_{1}(t)\|u(t)\|^{2}-\frac{1}{2} h_{2}(t)\|v(t)\|_{\Gamma}^{2} \\
& \left.-\frac{M_{3}}{2}\left(h_{1} \square u\right)(t)-\frac{M_{3}}{2}\left(h_{2} \square v\right)(t)-\left\|v^{\prime}(t)\right\|_{\Gamma}^{2}\right] .
\end{aligned}
$$

Therefore, by (5.16)-(5.22) we obtain

$$
\begin{aligned}
\psi^{\prime}(t) \leq & -e(t)+\frac{\beta_{2}}{4 \eta}\left\|\nabla u^{\prime}(t)\right\|^{2}+\frac{\beta_{3}}{4 \eta}\left\|\nabla v^{\prime}(t)\right\|^{2}-\left(\frac{1}{2}-\beta_{2} \eta\right)\|\nabla u(t)\|^{2} \\
& +\frac{1}{\gamma+2} h_{2}(t)\|v(t)\|_{\gamma+2, \Gamma}^{\gamma+2}-\frac{3}{4}\left(\|\nabla u(t)\|^{2}+\|\nabla v(t)\|^{2}\right)^{2}-\left(\frac{3}{2 \alpha_{1}}+\frac{\left(\mu_{1}+\mu_{2}\right) \alpha_{2}}{4 \eta}\right) \\
& \times\left[e^{\prime}(t)+\beta_{2}\left\|\nabla u^{\prime}(t)\right\|^{2}+\beta_{3}\left\|\nabla v^{\prime}(t)\right\|^{2}+\frac{\alpha_{3}}{2}\left\|v^{\prime}(t)\right\|_{\rho+2, \Gamma}^{\rho+2}+\xi_{1} h_{2}(t)\|v(t)\|_{\gamma+2, \Gamma}^{\gamma+2}\right. \\
& \left.+\frac{1}{2} h_{1}(t)\|u(t)\|^{2}+\frac{1}{2} h_{2}(t)\|v(t)\|_{\Gamma}^{2}+\frac{M_{3}}{2}\left(h_{1} \square u\right)(t)+\frac{M_{3}}{2}\left(h_{2} \square v\right)(t)+\left\|v^{\prime}(t)\right\|_{\Gamma}^{2}\right] \\
& -\left(\frac{\beta_{1}}{2}-\eta-a \frac{\sqrt{\mu_{1} \mu_{2}}}{2}\right)\|\Delta u(t)\|^{2}-\left(\frac{1}{2}-2 \eta-\beta_{3} \eta-a \frac{\sqrt{\mu_{1} \mu_{2}}}{2}\right)\|\nabla v(t)\|^{2}
\end{aligned}
$$




$$
\begin{aligned}
& +\left(h_{1} \square u\right)(t)+\left(h_{2} \square v\right)(t)-\frac{a}{2}\|u(t)\|^{2}-\frac{a}{2}\|v(t)\|^{2}-\frac{1}{2}\|v(t)\|_{\Gamma}^{2}+\|u(t)\|^{2} \int_{0}^{t} h_{1}(s) d s \\
& +\|v(t)\|_{\Gamma}^{2} \int_{0}^{t} h_{2}(s) d s+\frac{\mu_{3}^{2}}{4 \eta}\left\|v^{\prime}(t)\right\|_{\Gamma}^{2}+\theta(\eta)\left\|v^{\prime}(t)\right\|_{\rho+2, \Gamma}^{\rho+2}+\eta\|v(t)\|_{\rho+2, \Gamma}^{\rho+2} \\
\leq & -e(t)-R e^{\prime}(t)-\beta_{2}\left(R-\frac{1}{4 \eta}\right)\left\|\nabla u^{\prime}(t)\right\|^{2}-\beta_{3}\left(R-\frac{1}{4 \eta}\right)\left\|\nabla v^{\prime}(t)\right\|^{2} \\
& -\left(\frac{1}{2}-\beta_{2} \eta\right)\|\nabla u(t)\|^{2}-\left(\frac{\alpha_{3}}{2} R-\theta(\eta)\right)\left\|v^{\prime}(t)\right\|_{\rho+2, \Gamma}^{\rho+2} \\
& -\left(\xi_{1} R-\frac{1}{\gamma+2}\right) h_{2}(t)\|v(t)\|_{\gamma+2, \Gamma}^{\gamma+2}-\left(\frac{1}{2} R h_{1}(t)-\int_{0}^{t} h_{1}(s) d s\right)\|u(t)\|^{2} \\
& -\left(\frac{1}{2}+\frac{1}{2} R h_{2}(t)-\int_{0}^{t} h_{2}(s) d s\right)\|v(t)\|_{\Gamma}^{2}-\left(\frac{M_{3}}{2} R-1\right)\left(h_{1} \square u\right)(t) \\
& -\left(\frac{M_{3}}{2} R-1\right)\left(h_{2} \square v\right)(t)-\left(\frac{\beta_{1}}{2}-\eta-a \frac{\sqrt{\mu_{1} \mu_{2}}}{2}\right)\|\Delta u(t)\|^{2} \\
& -\left(\frac{1}{2}-2 \eta-\beta_{3} \eta-a \frac{\sqrt{\mu_{1} \mu_{2}}}{2}\right)\|\nabla v(t)\|^{2}-\left(R-\frac{\mu_{3}^{2}}{4 \eta}\right)\left\|v^{\prime}(t)\right\|_{\Gamma}^{2}+\eta\|v(t)\|_{\rho+2, \Gamma}^{\rho+2},
\end{aligned}
$$

where we denote $R=\left(3 / 2 \alpha_{1}+\left(\mu_{1}+\mu_{2}\right) \alpha_{2} / 4 \eta\right)$ for convenient. Hence from (5.8), the above inequality, and noticing $\gamma=\rho$, we get

$$
\begin{aligned}
e_{\varepsilon}^{\prime}(t)= & e^{\prime}(t)+\varepsilon \psi^{\prime}(t) \\
\leq & -\varepsilon e(t)-\beta_{2}\left(1-\frac{\varepsilon}{4 \eta}\right)\left\|\nabla u^{\prime}(t)\right\|^{2}-\beta_{3}\left(1-\frac{\varepsilon}{4 \eta}\right)\left\|\nabla v^{\prime}(t)\right\|^{2} \\
& -\left(\frac{\alpha_{3}}{2}-\varepsilon \theta(\eta)\right) h_{2}(t)\left\|v^{\prime}(t)\right\|_{\rho+2, \Gamma}^{\rho+2}-\varepsilon\left(\frac{1}{2}-\beta_{2} \eta\right)\|\nabla u(t)\|^{2} \\
& -\left(\xi_{1}-\varepsilon \frac{1}{\gamma+2}-\varepsilon \eta\right) h_{2}(t)\|v(t)\|_{\gamma+2, \Gamma}^{\gamma+2}-\left(\frac{1}{2} h_{1}(t)-\varepsilon \int_{0}^{t} h_{1}(s) d s\right)\|u(t)\|^{2} \\
& -\left(\frac{1}{2} h_{2}(t)-\varepsilon \int_{0}^{t} h_{2}(s) d s\right)\|v(t)\|_{\Gamma}^{2}-\left(\frac{M_{3}}{2}-\varepsilon\right)\left(h_{1} \square u\right)(t)-\left(\frac{M_{3}}{2}-\varepsilon\right)\left(h_{2} \square v\right)(t) \\
& -\varepsilon\left(\frac{1}{2}-2 \eta-\beta_{3} \eta-a \frac{\sqrt{\mu_{1} \mu_{2}}}{2}\right)\left(\|\Delta u(t)\|^{2}+\|\nabla v(t)\|^{2}\right) \\
& -\left(1-\varepsilon \frac{\mu_{3}^{2}}{4 \eta}\right)\left\|v^{\prime}(t)\right\|_{\Gamma}^{2},
\end{aligned}
$$

hence, if we denote

$$
\tilde{\varepsilon}=\min \left\{\frac{\alpha_{3}}{2 \theta(\eta)}, \frac{\xi_{1}(\gamma+2)}{1+(\gamma+2) \eta}, \frac{\left\|h_{1}\right\|_{L^{\infty}(0, \infty)}}{2\left\|h_{1}\right\|_{L^{1}(0, \infty)}}, \frac{\left\|h_{2}\right\|_{L^{\infty}(0, \infty)}}{2\left\|h_{2}\right\|_{L^{1}(0, \infty)}}, \frac{M_{3}}{2}, \frac{4 \eta}{\mu_{3}^{2}}, 4 \eta\right\}
$$


and choosing $\varepsilon \in(0, \tilde{\varepsilon}]$, we obtain

$$
e_{\varepsilon}^{\prime}(t) \leq-\varepsilon \xi_{3} e(t)
$$

for some constant $\xi_{3}>0$.

Proof of decay. Let us define $\widehat{\varepsilon}=\min \left\{1 / 2 \xi_{2}, \widetilde{\varepsilon}\right\}$ and consider $\varepsilon \in(0, \hat{\varepsilon}]$. From Lemma 5.1 we have

$$
\left(1-\xi_{2} \varepsilon\right) e(t) \leq e_{\varepsilon}(t) \leq\left(1+\xi_{2} \varepsilon\right) e(t)
$$

and so

$$
\frac{1}{2} e(t) \leq e_{\varepsilon}(t) \leq \frac{3}{2} e(t)
$$

From (5.28) we get

$$
-\varepsilon \xi_{3} e(t) \leq-\varepsilon \frac{2}{3} \xi_{3} e_{\varepsilon}(t) .
$$

Hence from (5.29) and Lemma 5.2, we obtain

$$
e_{\varepsilon}^{\prime}(t) \leq-\varepsilon \frac{2}{3} \xi_{3} e_{\varepsilon}(t) .
$$

That is,

$$
\frac{d}{d t}\left(e_{\varepsilon}(t) \exp \left\{\frac{2 \varepsilon}{3} \xi_{3} t\right\}\right) \leq 0 .
$$

Integrating the last inequality over $[0, t]$, we get

$$
e_{\varepsilon}(t) \leq e_{\varepsilon}(0) \exp \left\{-\frac{2 \varepsilon}{3} \xi_{3} t\right\} .
$$

From (5.28) and (5.32), we have

$$
e(t) \leq 3 e(0) \exp \left\{-\frac{2 \varepsilon}{3} \xi_{3} t\right\} .
$$

Hence, from (5.9) and (5.33) we obtain

$$
E(t) \leq \min \left\{l_{1}, l_{2}\right\}^{-1} e(t) \leq 3 e(0) \min \left\{l_{1}, l_{2}\right\}^{-1} \exp \left\{-\frac{2 \varepsilon}{3} \xi_{3} t\right\}, \quad t \geq t_{0}, \forall \varepsilon \in(0, \hat{\varepsilon}],
$$

that is,

$$
E(t) \leq C \exp (-\xi t) \quad \forall t \geq t_{0},
$$

where $C=3 e(0) \min \left\{l_{1}, l_{2}\right\}^{-1}$ and $\xi=(2 \varepsilon / 3) \xi_{3}$.

Therefore we have proved the exponential decay of solutions. 


\section{Acknowledgment}

The authors express gratitude to the anonymous referee for his/her helpful suggestions.

\section{References}

[1] M. Aassila, A note on the boundary stabilization of a compactly coupled system of wave equations, Applied Mathematics Letters 12 (1999), no. 3, 19-24.

[2] M. Aassila, M. M. Cavalcanti, and J. A. Soriano, Asymptotic stability and energy decay rates for solutions of the wave equation with memory in a star-shaped domain, SIAM Journal on Control and Optimization 38 (2000), no. 5, 1581-1602.

[3] J. J. Bae, On uniform decay of the solution for a damped nonlinear coupled system of wave equations with nonlinear boundary damping and memory term, Applied Mathematics and Computation 148 (2004), no. 1, 207-223.

[4] M. M. Cavalcanti, V. N. Domingos Cavalcanti, J. S. Prates Filho, and J. A. Soriano, Existence and exponential decay for a Kirchhoff-Carrier model with viscosity, Journal of Mathematical Analysis and Applications 226 (1998), no. 1, 40-60.

[5] __ Existence and uniform decay rates for viscoelastic problems with nonlinear boundary damping, Differential and Integral Equations 14 (2001), no. 1, 85-116.

[6] S. M. Choo and S. K. Chung, Finite difference approximate solutions for the strongly damped extensible beam equations, Applied Mathematics and Computation 112 (2000), no. 1, 11-32.

[7] V. Georgiev and G. Todorova, Existence of a solution of the wave equation with nonlinear damping and source terms, Journal of Differential Equations 109 (1994), no. 2, 295-308.

[8] A. Guesmia, Energy decay for a damped nonlinear coupled system, Journal of Mathematical Analysis and Applications 239 (1999), no. 1, 38-48.

[9] S. Jiang and J. E. Muñoz Rivera, A global existence theorem for the Dirichlet problem in nonlinear n-dimensional viscoelasticity, Differential and Integral Equations 9 (1996), no. 4, 791-810.

[10] V. Komornik and P. Loreti, Ingham-type theorems for vector-valued functions and observability of coupled linear systems, SIAM Journal on Control and Optimization 37 (1999), no. 2, 461-485.

[11] V. Komornik and E. Zuazua, A direct method for the boundary stabilization of the wave equation, Journal de Mathématiques Pures et Appliquées. Neuvième Série 69 (1990), no. 1, 33-54.

[12] T. F. Ma, Existence results and numerical solutions for a beam equation with nonlinear boundary conditions, Applied Numerical Mathematics 47 (2003), no. 2, 189-196.

[13] Z. Oniszczuk, Free transverse vibrations of an elastically connected rectangular plate-membrane complex system, Journal of Sound and Vibration 264 (2003), no. 1, 37-47.

[14] R. E. Showalter, Monotone operators in Banach space and nonlinear partial differential equations, Mathematical Surveys and Monographs, vol. 49, American Mathematical Society, Rhode Island, 1997.

[15] A. Soufyane, Uniform stability of displacement coupled second-order equations, Electronic Journal of Differential Equations 2001 (2001), no. 25, 1-10.

[16] E. Vitillaro, Global existence for the wave equation with nonlinear boundary damping and source terms, Journal of Differential Equations 186 (2002), no. 1, 259-298.

Haihong Liu: Department of Mathematical Sciences, Tsinghua University, Beijing 100084, China E-mail address:1hh02@mails.tsinghua.edu.cn

Ning Su: Department of Mathematical Sciences, Tsinghua University, Beijing 100084, China

E-mail address: nsu@math.tsinghua.edu.cn 


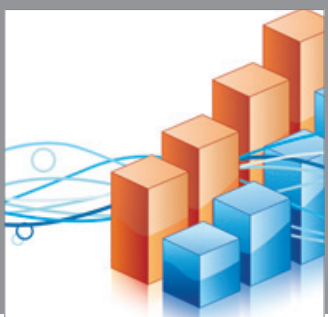

Advances in

Operations Research

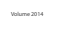

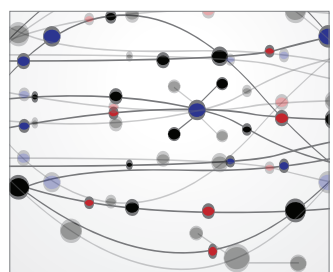

\section{The Scientific} World Journal
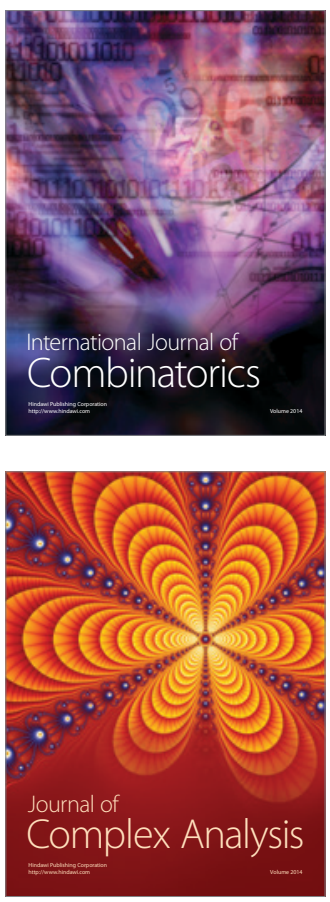

International Journal of

Mathematics and

Mathematical

Sciences
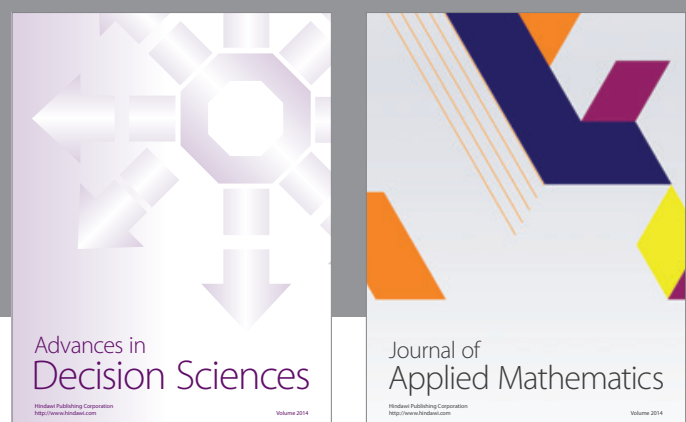

Journal of

Applied Mathematics
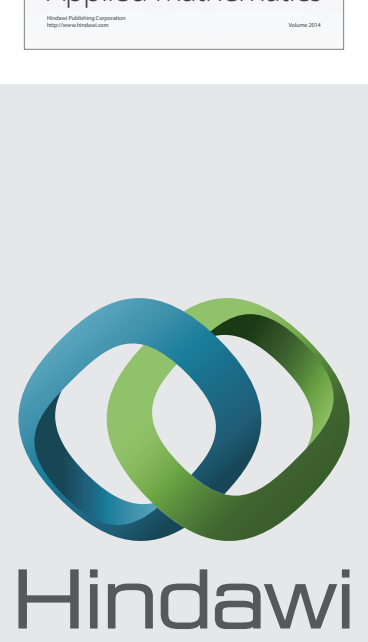

Submit your manuscripts at http://www.hindawi.com
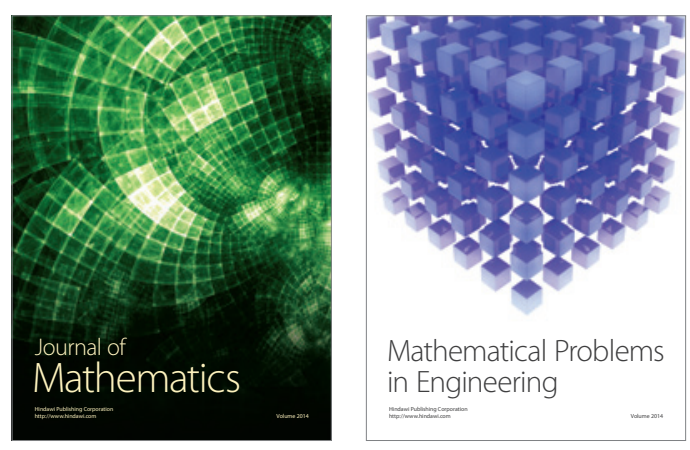

Mathematical Problems in Engineering
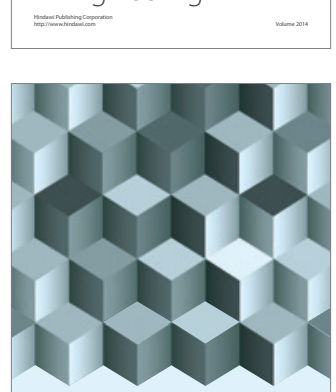

Journal of

Function Spaces
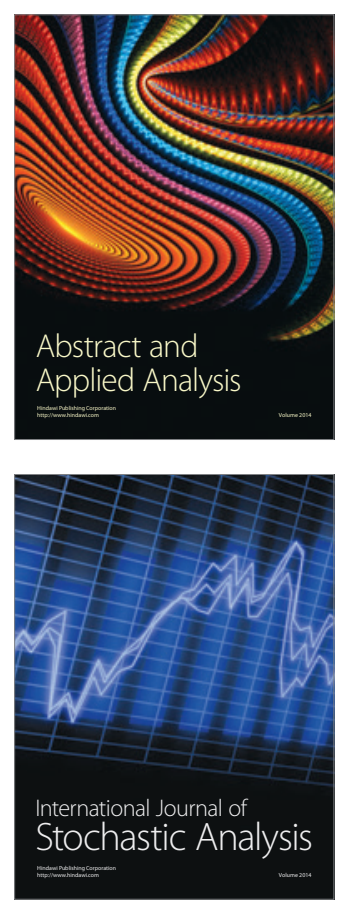

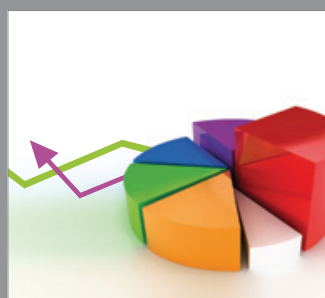

ournal of

Probability and Statistics

Promensencen
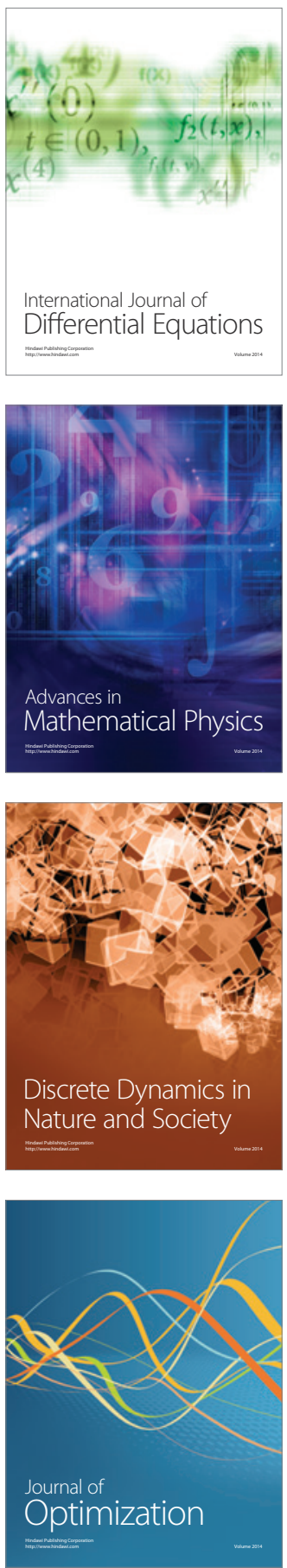